\title{
Stability and instability of solitary waves of the fifth-order KdV equation: a numerical framework
}

\author{
Thomas J. Bridges, Gianne Derks and Georg Gottwald \\ Department of Mathematics and Statistics, \\ University of Surrey, Guildford, Surrey, GU2 7XH, UK
}

July 24, 2002

\begin{abstract}
The spectral problem associated with the linearization about solitary waves of the generalized fifth-order $\mathrm{KdV}$ equation is formulated in terms of the Evans function, a complex analytic function whose zeros correspond to eigenvalues. A numerical framework, based on a fast robust shooting algorithm on exterior algebra spaces is introduced. The complete algorithm has several new features, including a rigorous numerical algorithm for choosing starting values, a new method for numerical analytic continuation of starting vectors, the role of the Grassmannian $G_{2}\left(\mathbb{C}^{5}\right)$ in choosing the numerical integrator, and the role of the Hodge star operator for relating $\Lambda^{2}\left(\mathbb{C}^{5}\right)$ and $\Lambda^{3}\left(\mathbb{C}^{5}\right)$ and deducing a range of numerically computable forms for the Evans function. The algorithm is illustrated by computing the stability and instability of solitary waves of the fifth-order $\mathrm{KdV}$ equation with polynomial nonlinearity.
\end{abstract}

\section{Table of Contents}

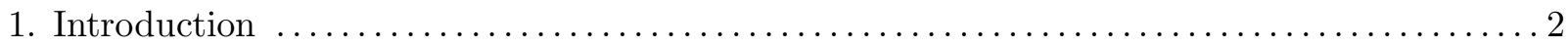

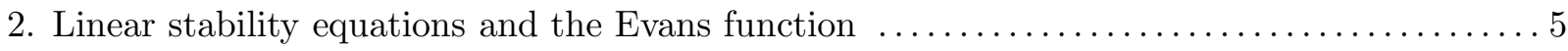

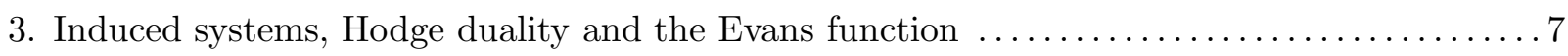

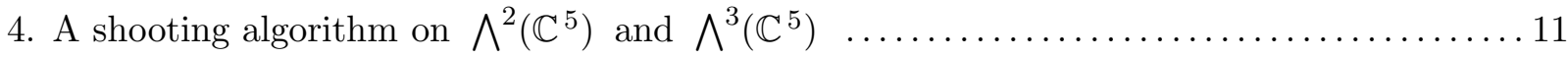

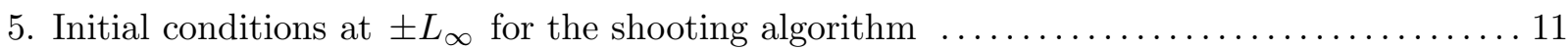

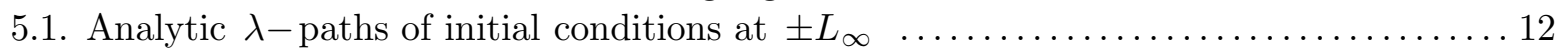

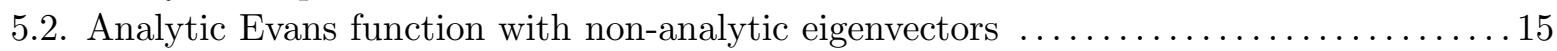

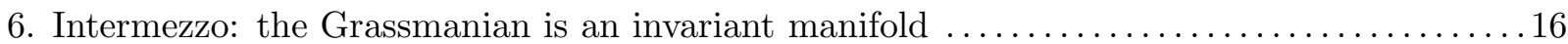

6.1. Can the Grassmannian be more attractive ? .............................. 17

7. Details of the system at infinity for linearized 5 th-order $\mathrm{KdV} \ldots \ldots \ldots \ldots \ldots \ldots \ldots \ldots$

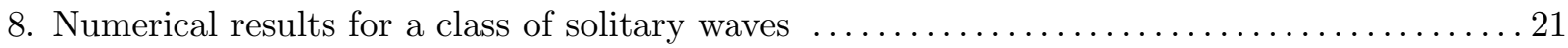

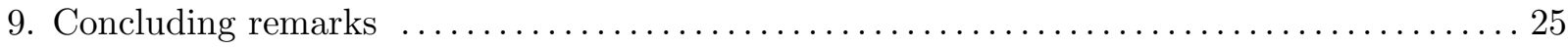

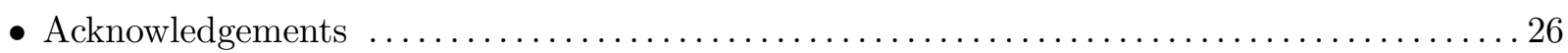

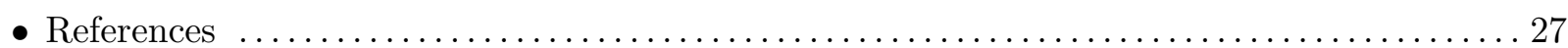




\section{Introduction}

The fifth-order $\mathrm{KdV}$ equation is a model equation for plasma waves, capillary-gravity water waves, and other dispersive phenomena when the cubic KdV-type dispersion is weak. Such equations can be written in the general form

$$
\frac{\partial u}{\partial t}+\alpha \frac{\partial^{3} u}{\partial x^{3}}+\beta \frac{\partial^{5} u}{\partial x^{5}}=\frac{\partial}{\partial x} f\left(u, u_{x}, u_{x x}\right),
$$

for the scalar-valued function $u(x, t)$, where $\alpha$ and $\beta$ are real parameters with $\beta \neq 0$ and $f\left(u, u_{x}, u_{x x}\right)$ is some smooth function.

The form of (1.1) which occurs most often in applications is with $f\left(u, u_{x}, u_{x x}\right)=K u^{p+1}$ where $K$ is a nonzero constant and $p \geq 1$ generally an integer. This equation first appears in the literature in the work of HASIMOTO and KAWAHARA with $p=1$ where generalized solitary waves are computed numerically [37]. Motivated by water waves, model equations with a larger class of nonlinearities are derived by CRAIG \& Groves [22]. Other forms for (1.1) with further generalization of $f$ appear in $[30,39,40]$.

The solutions of (1.1) of greatest interest in applications are travelling solitary waves. Such states, travelling at speed $c$ and of the form $u(x, t)=\hat{u}(x-c t)$, satisfy the fourth-order nonlinear differential equation

$$
\beta \hat{u}_{x x x x}+\alpha \hat{u}_{x x}-2 c \hat{u}-f\left(\hat{u}, \hat{u}_{x}, \hat{u}_{x x}\right)=A,
$$

where $A$ is a constant of integration. This system is not integrable in general, and can have an extraordinary range of solitary waves. A review of the known classes is given by ChAmpNeys[18].

However, there is very little in the literature about the stability of these solitary waves. When the PDE is Hamiltonian, for example when $f\left(u, u_{x}, u_{x x}\right)$ is a gradient function, one can appeal to energy-momentum arguments for nonlinear stability (e.g. ILL'IChev \& SEmenov [33], Karpman [35], Dey, Khare \& Kumar [23], Dias \& Kuznetsov [24], Levandosky [40]), and the symplectic Evans matrix for a range of analytical techniques for linear instability (BRIDGES \& DeRKS $[13,14])$. However, the energy momentum method requires the second variation of the main functional to have a precise eigenvalue structure which is often violated. The symplectic Evans matrix provides a geometric theory for analytic prediction of instability of solitary waves of (1.1) [13], but these methods do not apply when $f$ in (1.1) is non-gradient. On the other hand, it would be useful to have a numerical framework for (1.1) even in the Hamiltonian case.

In the non-Hamiltonian case, the only known general approach is the Evans function framework. This function can be constructed for the linearization about a solitary wave of the 5th-order $\mathrm{KdV}$ equation (as long as the solitary wave exists), but there are no results in the literature on the construction or analysis of the Evans function for (1.1), except in the Hamiltonian case [14].

The spectral problem for the linearization about a solitary wave can also be formulated numerically without consideration of the Evans function. For example, BEYN \& LoREnZ [10] consider a linearized complex Ginzburg-Landau equation, Barashenkov, Pelinovsky \& Zemlyanaya [7] and Barashenkov \& Zemlyanaya [8] consider a linearized nonlinear Schrödinger system, and Liefvendahl \& Kreiss [41] study the stability of viscous shock profiles. In all three cases, they approach the problem by discretizing the spectral problem on the truncated domain $x \in\left[-L_{\infty}, L_{\infty}\right]$ using finite differences, collocation or a spectral method, reducing it to a very large matrix eigenvalue problem. There are two central difficulties with this approach. First, in general the exact asymptotic boundary conditions at $x= \pm L_{\infty}$ depend on $\lambda$ in a nonlinear way, and so application of the exact asymptotic boundary conditions changes the problem to a nonlinear in the parameter matrix eigenvalue problem, in which case matrix eigenvalue solvers can no longer be used. In all the above cases, artificial boundary conditions such as Dirichlet or periodic boundary conditions, were applied, in order to retain linearity in the spectral parameter. 
Secondly, the approximate boundary conditions lead to spurious discrete eigenvalues generated from the fractured continuous spectrum. If the continuous spectrum is strongly stable (that is, the continuous spectrum is stable and there is a gap between the continuous spectrum and the imaginary axis) this does not normally generate spurious unstable eigenvalues. However, if the continuous spectrum lies on the imaginary axis, spurious eigenvalues may be emitted into the unstable half plane. Indeed, BARASHENKOV \& ZEMLYANAYA [8] give an extreme example, where a large number of spurious unstable eigenvalues are generated by the matrix discretization (see Figure 1 of [8]).

An example of the significance of using exact asymptotic boundary conditions is Keller's result on systems with the "constant tail property". If $\mathbf{A}(x, \lambda)$ is constant for $x>x_{0}$, then the finite domain problem, $x \in\left[-L_{\infty}, L_{\infty}\right]$, recovers the spectrum of the infinite domain problem exactly, when the correct asymptotic boundary conditions are used (cf. KELLER [38] $\S 4.2$ and Theorem 4.26). Although exponential decay of $\mathbf{A}(x, \lambda)$ to $\mathbf{A}_{\infty}(\lambda)$ will not result in exact replication of the spectrum, the result of Keller is strongly suggestive that the approximation will be much better behaved.

The linearization of (1.1) about a basic solitary wave leads to a system of the form

$$
\mathbf{v}_{x}=\mathbf{A}(x, \lambda) \mathbf{v}, \quad \mathbf{v} \in \mathbb{C}^{5}
$$

where $\lambda \in \mathbb{C}$ is the spectral parameter and $\mathbf{A}(x, \lambda)$ is a matrix in $\mathbb{C}^{5 \times 5}$, whose limit for $x \rightarrow \infty$ exists.

The purpose of this paper is to construct the Evans function for the linearization about any solitary wave satisfying (1.2), with exponential decay to zero as $x \rightarrow \pm \infty$, and to introduce a numerical framework to compute this Evans function. One advantage of the Evans function is that the exact asymptotic boundary conditions are built into the definition in an analytic way.

The Evans function is a complex analytic function associated with (1.3) whose zeros correspond to eigenvalues of the spectral problem associated with the linearization about a solitary wave solution. It was first introduced by Evans [26] and generalized by AlExander, Gardner $\&$ Jones [3]. The details of its construction for (1.3) are given in $\S 3$. Crucial to the construction is the number of negative eigenvalues of the 'system at infinity', that is, the matrix $\mathbf{A}_{\infty}(\lambda)$ which is associated with the limit as $x \rightarrow \infty$ of $\mathbf{A}(x, \lambda)$. It is assumed that the number of negative eigenvalues is constant for $\lambda \in \Lambda$, where $\Lambda$ is a simply-connected subset of $\mathbb{C}$. Let $k$ be the number of negative eigenvalues of $\mathbf{A}_{\infty}(\lambda)$ for $\lambda \in \Lambda$.

The first numerical computation of the Evans function was by Evans himself in Evans \& Feroe [27]. This work was followed by Swinton \& Elgin [47] and Pego, Smereka \& WeinSTEIN [45]. However, in all three papers $k=1$, in which case a standard shooting argument can be used (i.e. numerical exterior algebra is not needed). This approach will fail if $k>1$, which is a case that arises commonly for the linearized 5th-order KdV.

A naive approach would be to take the $k$ eigenvectors - associated with the $k$ eigenvalues of negative real part - as starting vectors for the integration of (1.3) from $x=L_{\infty}$ to $x=0$, with a similar strategy for $x<0$. This approach will fail for two reasons. Firstly, although the $k$ solutions are linearly independent for the continuous problem, they will not maintain linear independence numerically, because all vectors will be attracted to the eigenvector associated with the largest growth rate. This is a classic numerical problem of stiffness and the standard approach to resolving this difficulty is to use discrete or continuous orthogonalization. However, orthogonalization will firstly cause problems with analyticity (i.e. taking the length of a vector which depends analytically on a parameter, is a non-analytic operation), secondly orthogonalization transforms the linear equation to a nonlinear equation.

The second more subtle problem with integrating $k$ individual vectors is that the starting eigenvectors will not in general be analytic for all $\lambda$ in a given open set. For distinguished values of $\lambda$ the eigenvalues of $\mathbf{A}_{\infty}(\lambda)$ may coalesce, resulting in branch points in the complex $\lambda$ plane. 
All the above problems are eliminated by using exterior algebra. The idea of integrating on the exterior algebra of $\mathbb{C}^{n}$ has its origins in the compound matrix method introduced by $\mathrm{NG}$ \& REID [44] for hydrodynamic stability problems. This method is widely used to integrate the Orr-Sommerfeld equation in hydrodynamic stability (cf. Drazin \& REID [25], BRIDGES [11]). In [11], it was first pointed out that exterior algebra gives a coordinate-free formulation of the compound matrix method, and that the compound matrix method is an example of a Grassmannian integrator. In other words, fundamentally, the solution paths do not lie in a linear space, but correspond to paths on $G_{k}\left(\mathbb{C}^{n}\right)$. This latter property changes the nature of the numerical integration, requiring methods which preserve the manifold of the induced system of ODEs (see $\S 6$ herein).

Exterior algebra provides a coordinate-free formulation of compound matrices and a wider range of tools for integrating ODEs on infinite domains. The compound matrix method is the special case where Plücker coordinates are used. The general theory for integrating ODEs on $\mathbb{C}^{n}$ with $k$-dimensional preferred subspaces - of which the linearization about solitary waves is a special case - including issues such as boundary conditions, analyticity, automated construction of induced systems, the role of Hodge duality, and a range of examples, is given in AlLEN \& BRIDGES [5].

Numerical exterior algebra or the compound matrix method gives a framework for extending the computation of the Evans function to the case $k>1$. This was first done by PEgo (see Appendix II of Alexander \& SACHS [4]), where a form of the compound matrix method was used to compute the Evans function for the linearization about solitary waves of the Boussinesq equation. In AfENDIKOv \& BRIDGes [2], the Evans function for the linearization about solitary waves of the complex Ginzburg-Landau equation was formulated, where $k=2$ and the dimension of the ODE is $n=4$, and a numerical scheme based on the compound matrix method was used to compute unstable eigenvalues associated with the Hocking-Stewartson pulse. In [2] a numerical scheme which preserved the Grassmannian $G_{2}\left(\mathbb{C}^{4}\right)$ exactly was used.

Independently, BRIN [16] introduced a numerical framework for computing the Evans function based on exterior algebra and a numerical implementation of Kato's Theorem (BRIN \& ZUMBRUN [17]). Numerical results for the case $k=2$ and $n=4$, for the Evans function associated with the linearization about viscous shock profiles, are presented.

The case $k=2$ and $n=4$ has some nice properties, the most important of which is that the Grassmannian $G_{2}\left(\mathbb{C}^{4}\right)$ is defined by a single quadric [5], and the characteristic polynomical associated with the system at infinity is described by a quartic, and so the roots can be found analytically.

The case $k=2$ and $n=5$, which is central to the study of (1.3), has never been considered and brings in new problems: the Grassmannian $G_{2}\left(\mathbb{C}^{5}\right)$ is defined by five non-independent quadrics, and the characteristic polynomial associated with the system at infinity is quintic and so will require numerical solution in general. The system at infinity generates starting vectors which are required to be analytic. A new algorithm for numerical analytic continuation is proposed in $§ 5.1$.

Given analytic starting vectors at $x=L_{\infty}$, the numerical strategy is to integrate the induced system associated with (1.3) from $x=L_{\infty}$ to $x=0$ on $\wedge^{2}\left(\mathbb{C}^{5}\right)$, and to integrate the induced system associated with (1.3) from $x=-L_{\infty}$ to $x=0$ on $\bigwedge^{3}\left(\mathbb{C}^{5}\right)$. These solutions are then matched at $x=0$ to give a numerical expression for the Evans function. To make this matching rigorous, the Hodge star operator, which is the natural isomorphism between $\bigwedge^{2}\left(\mathbb{C}^{5}\right)$ and $\bigwedge^{3}\left(\mathbb{C}^{5}\right)$, is used. The Hodge star operator preserves linearity, decomposability and analyticity (in the sense that it appears here, when complex conjugation in an inner product is composed with Hodge star). Hodge star is the backbone of the argument used to simplify the integration for $x<0$, by bringing in the adjoint on $\bigwedge^{2}\left(\mathbb{C}^{5}\right)$ in a geometric way, and it provides several computable formulae for the Evans function.

The algorithm is quite general, and applies to any given solitary wave of (1.2), whether an 
analytic expression or given numerically. It is demonstrated by computing the stability and instability - as a function of $p$ - for the nonlinearity $f=K u^{p+1}$ where $K$ is a constant and $p$ is a positive real number.

\section{Linear stability equations and the Evans function}

Suppose there exists a solitary wave of (1.1) of the form $u(x, t)=\widehat{u}(x-c t)$, i.e., $\widehat{u}(x)$ satisfies $(1.2)$, and that the solitary wave decays exponentially as $x \rightarrow \pm \infty$ to zero (various generalizations of this condition are possible but are not considered here). Linearizing (1.1) about this basic solitary wave results in the PDE

$$
\hat{v}_{t}-c \hat{v}_{x}+\alpha \hat{v}_{x x x}+\beta \hat{v}_{x x x x x}=\frac{\partial}{\partial x}\left[f_{1}(x) \hat{v}+f_{2}(x) \hat{v}_{x}+f_{3}(x) \hat{v}_{x x}\right],
$$

where

$$
f_{1}(x)=\left.\frac{\partial}{\partial u} f\left(u, u_{x}, u_{x x}\right)\right|_{u=\hat{u}(x)}, \quad f_{2}(x)=\left.\frac{\partial}{\partial u_{x}} f\left(u, u_{x}, u_{x x}\right)\right|_{u=\hat{u}(x)}
$$

and

$$
f_{3}(x)=\left.\frac{\partial}{\partial u_{x x}} f\left(u, u_{x}, u_{x x}\right)\right|_{u=\hat{u}(x)} .
$$

With the spectral ansatz $\hat{v}=e^{\lambda t} v$, the resulting spectral problem is

$$
\mathbf{L} v=\lambda v, \quad v \in \mathcal{D}(\mathbf{L}) \subset \mathbb{X},
$$

where

$$
\mathbf{L} v:=\left[f_{1}(x) v+f_{2}(x) v_{x}+f_{3}(x) v_{x x}\right]_{x}-\beta v_{x x x x x}-\alpha v_{x x x}+c v_{x},
$$

$\mathcal{D}(\mathbf{L})$ is the domain of $\mathbf{L}$, and $\mathbb{X}$ is some suitably chosen function space such as $L_{2}(\mathbb{R})$. A point $\lambda \in \mathbb{C}$ is an eigenvalue of $\mathbf{L}$, denoted $\lambda \in \sigma_{p}(\mathbf{L})$, if there exists a pair $(v, \lambda) \in(\mathcal{D}(\mathbf{L}), \mathbb{C})$ satisfying (2.1).

Define

$$
\mathbb{C}^{+}=\{\lambda \in \mathbb{C}: \Re(\lambda)>0\} .
$$

The basic solitary wave is said to be spectrally unstable if there is at least one value of $\lambda$ in $\sigma_{p}(\mathbf{L}) \cap \mathbb{C}^{+}$. It is weakly spectrally stable if $\sigma_{p}(\mathbf{L}) \cap \mathbb{C}^{+}$is empty.

While in finite-dimensional Hamiltonian systems weak spectral stability implies spectral stability, in infinite dimensions the issue is more subtle. For example, instability can be created by resonance between discrete neutral modes and neutral modes in the continuous spectrum (cf. Soffer \& Weinstein [46]). We use the qualifier weak to emphasize that spectral activity on the imaginary axis is not considered, and to remind that neutral spectra can impact a conclusion of "spectral stability".

We will assume that the essential spectrum, denoted by $\sigma_{c}(\mathbf{P})$, is not unstable. This hypothesis reduces to $\lim _{x \rightarrow \pm \infty} f_{2}(x) \geq 0$. To see this, let $f_{j}^{\infty}=\lim _{x \rightarrow \pm \infty} f_{j}(x)$, then

$$
\sigma_{c}(\mathbf{L})=\left\{\lambda \in \mathbb{C}: \lambda=\mathrm{i} k\left(c+f_{1}^{\infty}-\beta k^{4}+\alpha k^{2}-k^{2} f_{3}^{\infty}\right)-k^{2} f_{2}^{\infty}, k \in \mathbb{R}\right\},
$$

and so $\sigma_{c}(\mathbf{L}) \cap \mathbb{C}^{+}$is empty if $f_{2}^{\infty} \geq 0$.

In this paper a numerical scheme will be introduced which will discriminate between spectral instability and weak spectral stability of eigenvalues, based on the Evans function, for the 
fifth-order KdV. Therefore we will be looking for eigenvalues in $\mathbb{C}^{+}$, away from the continuous spectrum. The Evans function can be generalized to include analytic continuation through the continuous spectrum (cf. Gardner \& Zumbrun [28], Kapitula \& Sandstede [34]). Such a generalization can be used to find eigenvalues embedded in the continuous spectrum, but since the continuous spectrum is not unstable here, by hypothesis, this case will not be considered.

The Evans function associated with the linearized fifth order $\mathrm{KdV}$ is constructed as follows. The spectral problem (2.1) can be reformulated as the $\lambda$-dependent first-order system on the real line,

$$
\mathbf{v}_{x}=\mathbf{A}(x, \lambda) \mathbf{v}, \quad \mathbf{v} \in \mathbb{C}^{5},
$$

by taking

$$
\mathbf{v}=\left(v, v_{x}, v_{x x}, v_{x x x}, v_{5}\right), \quad \beta v_{5}=\beta v_{x x x x}+\left(\alpha-f_{3}\right) v_{x x}-f_{2} v_{x}-\left(c+f_{1}\right) v,
$$

leading to

$$
\mathbf{A}(x, \lambda)=\left(\begin{array}{ccccc}
0 & 1 & 0 & 0 & 0 \\
0 & 0 & 1 & 0 & 0 \\
0 & 0 & 0 & 1 & 0 \\
\frac{\left(c+f_{1}(x)\right)}{\beta} & \frac{f_{2}(x)}{\beta} & \frac{\left(-\alpha+f_{3}(x)\right)}{\beta} & 0 & 1 \\
-\frac{\lambda}{\beta} & 0 & 0 & 0 & 0
\end{array}\right) .
$$

Note that $\operatorname{Tr}(\mathbf{A}(x, \lambda))=0$. The matrix $\mathbf{A}(x, \lambda)$ has the asymptotic property that

$$
\lim _{x \rightarrow \pm \infty} \mathbf{A}(x, \lambda)=\mathbf{A}_{\infty}(\lambda)=\left(\begin{array}{ccccc}
0 & 1 & 0 & 0 & 0 \\
0 & 0 & 1 & 0 & 0 \\
0 & 0 & 0 & 1 & 0 \\
\rho_{1} & \rho_{2} & \rho_{3} & 0 & 1 \\
-\frac{\lambda}{\beta} & 0 & 0 & 0 & 0
\end{array}\right),
$$

where

$$
\rho_{1}=\frac{1}{\beta}\left(f_{1}^{\infty}+c\right), \quad \rho_{2}=\frac{1}{\beta} f_{2}^{\infty} \quad \text { and } \quad \rho_{3}=\frac{1}{\beta}\left(f_{3}^{\infty}-\alpha\right) .
$$

The characteristic polynomial of $\mathbf{A}_{\infty}(\lambda)$ is

$$
\Delta(\mu, \lambda)=\operatorname{det}\left[\mu \mathbf{I}-\mathbf{A}_{\infty}(\lambda)\right]=\mu^{5}-\rho_{3} \mu^{3}-\rho_{2} \mu^{2}-\rho_{1} \mu+\lambda / \beta .
$$

We will show later that for all $\lambda \in \Lambda$, where $\Lambda$ is a suitably defined subset of $\mathbb{C}^{+}$, the spectrum of $\mathbf{A}_{\infty}(\lambda)$ has $k$ eigenvalues with negative real part and $5-k$ eigenvalues with positive real part, where $k=1,2,3$ or $k=4$. The cases $k=1$ and $k=4$ are dual (and lead to an equivalent numerical formulation), and the cases $k=2$ and $k=3$ are dual. Numerically, the case $k=1$ is relatively straightforward (exterior algebra is not needed, and standard numerical integration is possible, as in $[27,45,47])$, and therefore we will concentrate on the case $k=2$.

The system (2.3) and the properties of the system at infinity, $\mathbf{A}_{\infty}(\lambda)$, are in standard form for the dynamical systems formulation of the spectral problem proposed by Evans [26] and generalized by Alexander, GARDNer \& Jones [3]. Let $\mathbf{U}^{+}(x, \lambda)$ represent the $k$-dimensional 
space of solutions of (2.3) which do not grow exponentially as $x \rightarrow+\infty$. Let $\mathbf{U}^{-}(x, \lambda)$ represent the $(5-k)$-dimensional space of solutions which decays as $x \rightarrow-\infty$. A value of $\lambda \in \Lambda$ is an eigenvalue if these two subspaces have a nontrivial intersection, and the Evans function determines if there is an intersection. The Evans function is defined by

$$
E(\lambda)=\mathrm{e}^{-\int_{0}^{x} \tau(s, \lambda) d s} \mathbf{U}^{-}(x, \lambda) \wedge \mathbf{U}^{+}(x, \lambda), \quad \lambda \in \Lambda,
$$

where $\wedge$ is the wedge product and

$$
\tau(x, \lambda)=\operatorname{Tr}(\mathbf{A}(x, \lambda)) .
$$

For the case of the fifth-order $\mathrm{KdV}$ this expression simplifies, since $\operatorname{Tr}(\mathbf{A}(x, \lambda))=0$, see (2.4).

In developing a numerical framework, the first issue is the construction of $\mathbf{U}^{+}(x, \lambda)$ and $\mathbf{U}^{-}(x, \lambda)$. They can be considered as paths in $\Lambda^{k}\left(\mathbb{C}^{n}\right)$ and $\bigwedge^{(n-k)}\left(\mathbb{C}^{n}\right)$ respectively. To calculate these paths, we will integrate the induced linear systems on $\bigwedge^{k}\left(\mathbb{C}^{n}\right)$ and $\bigwedge^{(n-k)}\left(\mathbb{C}^{n}\right)$ associated with (2.3). The Hodge star operator is an isomorphism between $\bigwedge^{k}\left(\mathbb{C}^{n}\right)$ and $\bigwedge^{(n-k)}\left(\mathbb{C}^{n}\right)$. It can be used to give an explicit numerical expression for the Evans function which is readily computable. However, the Hodge star operator can also be used to relate the system on $\bigwedge^{(n-k)}\left(\mathbb{C}^{n}\right)$ to the adjoint system on $\bigwedge^{k}\left(\mathbb{C}^{n}\right)$. This leads to a different expression of the Evans function, which is readily computable numerically and involves the integration of the system and its adjoint on $\bigwedge^{k}\left(\mathbb{C}^{n}\right)$. It is this expression that will be used in our numerical algorithm.

\section{Induced systems, Hodge duality and the Evans function}

Consider the linear system

$$
\mathbf{u}_{x}=\mathbf{A u}, \quad \mathbf{u} \in \mathbb{C}^{n} .
$$

A $k$-form is decomposable if it can be written as a pure form: a wedge product between $k$ linearly independent vectors in $\mathbb{C}^{n}$. Since every element in $\Lambda^{k}\left(\mathbb{C}^{n}\right)$ is a sum of decomposable elements, the linear system (3.1) induces a system on $\bigwedge^{k}\left(\mathbb{C}^{n}\right)$ :

$$
\mathbf{U}_{x}=\mathbf{A}^{(k)} \mathbf{U}, \quad \mathbf{U} \in \bigwedge^{k}\left(\mathbb{C}^{n}\right) .
$$

Here $\mathbf{A}^{(k)}$ is defined on a decomposable $k$-form $\mathbf{u}_{1} \wedge \cdots \wedge \mathbf{u}_{k}, \mathbf{u}_{i} \in \mathbb{C}^{n}$, as

$$
\mathbf{A}^{(k)}\left(\mathbf{u}_{1} \wedge \cdots \wedge \mathbf{u}_{k}\right):=\sum_{j=1}^{k} \mathbf{u}_{1} \wedge \cdots \wedge \mathbf{A} \mathbf{u}_{j} \wedge \cdots \wedge \mathbf{u}_{k}
$$

and extends by linearity to the non-decomposable elements in $\bigwedge^{k}\left(\mathbb{C}^{n}\right)$. This construction can be carried out in a coordinate free way, and general aspects of the numerical implementation of this theory can be found in Allen \& Bridges [5].

Let $\langle\cdot, \cdot\rangle_{n}$ be a complex inner product in $\mathbb{C}^{n}$. To construct an inner product on $\bigwedge^{k}\left(\mathbb{C}^{n}\right)$, let

$$
\mathbf{U}=\mathbf{u}_{1} \wedge \cdots \wedge \mathbf{u}_{k} \quad \text { and } \quad \mathbf{V}=\mathbf{v}_{1} \wedge \cdots \wedge \mathbf{v}_{k}, \quad \mathbf{u}_{i}, \mathbf{v}_{j} \in \mathbb{C}^{n}, \quad \forall i, j=1, \ldots, k,
$$

be any decomposable $k$-forms. The inner product of $\mathbf{U}$ and $\mathbf{V}$ is defined by

$$
\llbracket \mathbf{U}, \mathbf{V} \rrbracket_{k}:=\operatorname{det}\left[\begin{array}{ccc}
\left\langle\mathbf{u}_{1}, \mathbf{v}_{1}\right\rangle & \cdots & \left\langle\mathbf{u}_{1}, \mathbf{v}_{k}\right\rangle \\
\vdots & \ddots & \vdots \\
\left\langle\mathbf{u}_{k}, \mathbf{v}_{1}\right\rangle & \cdots & \left\langle\mathbf{u}_{k}, \mathbf{v}_{k}\right\rangle
\end{array}\right], \quad \mathbf{U}, \mathbf{V} \in \bigwedge^{k}\left(\mathbb{C}^{n}\right)
$$


Since every element in $\bigwedge^{k}\left(\mathbb{C}^{n}\right)$ is a sum of decomposable elements, this definition extends by linearity to any $k$-form in $\bigwedge^{k}\left(\mathbb{C}^{n}\right)$.

Both $\bigwedge^{k}\left(\mathbb{C}^{n}\right)$ and $\bigwedge^{(n-k)}\left(\mathbb{C}^{n}\right)$ are $d=\left(\begin{array}{l}n \\ k\end{array}\right)$ dimensional vector spaces, which are isomorphic and the isomorphism is the Hodge star operator. Details of the definition of the Hodge star operator in the complex case can be found in Chapter V of Wells [49]. To fix the orientation, choose a volume form $\mathcal{V}$. Hodge star, $\star: \bigwedge^{(n-k)}\left(\mathbb{C}^{n}\right) \rightarrow \bigwedge^{k}\left(\mathbb{C}^{n}\right)$, is defined by

$$
\llbracket \star \mathbf{W}, \mathbf{U} \rrbracket_{k} \mathcal{V}=\mathbf{W} \wedge \mathbf{U}, \quad \text { for any } \quad \mathbf{U} \in \bigwedge^{k}\left(\mathbb{C}^{n}\right), \mathbf{W} \in \bigwedge^{(n-k)}\left(\mathbb{C}^{n}\right) .
$$

Note that the action of Hodge star includes complex conjugation. If $\mathbf{W} \in \bigwedge^{(n-k)}\left(\mathbb{C}^{n}\right)$ is holomorphic then $\star \mathbf{W} \in \bigwedge^{k}\left(\mathbb{C}^{n}\right)$ is anti-holomorphic. Therefore $\overline{\star \mathbf{W}}$ is holomorphic (analytic).

In $[12,5]$ it is shown that if $\mathbf{W}(x) \in \bigwedge^{(n-k)}\left(\mathbb{C}^{n}\right)$ is a solution of $\mathbf{W}_{x}=\mathbf{A}^{(n-k)}(x) \mathbf{W}$, then $\star \mathbf{W} \in \bigwedge^{k}\left(\mathbb{C}^{n}\right)$ satisfies the differential equation

$$
\frac{d}{d x}(\star \mathbf{W})=\left[\overline{\tau(x)} \mathbf{I}_{d}-\left[\mathbf{A}^{(k)}(x)\right]^{*}\right](\star \mathbf{W}),
$$

where $\tau(x)=$ Trace $(\mathbf{A})$ and $\mathbf{I}_{d}$ is the identity operator on $\bigwedge^{k}\left(\mathbb{C}^{n}\right)$. Here and throughout the paper * denotes adjoint with respect to the ambient inner product, and it includes complex conjugation. A superscript $T$ will be used in situations where transpose without conjugation is implied. Defining

$$
\mathbf{V}^{-}(x, \lambda)=\mathrm{e}^{-\int_{0}^{x} \tau(s, \lambda) d s \overline{\star \mathbf{U}^{-}(x, \lambda)}}
$$

and noting that $\mathbf{V}^{-}(x, \lambda)$ is analytic, we find that $\mathbf{V}^{-}(x, \lambda)$ satisfies

$$
\frac{d}{d x} \mathbf{V}^{-}=-\left[\mathbf{A}^{(k)}(x)\right]^{T} \mathbf{V}^{-} .
$$

In other words, it is not necessary to integrate the induced system on $\bigwedge^{(n-k)}\left(\mathbb{C}^{n}\right)$; instead, for $x \leq 0$ the adjoint of the induced system on $\bigwedge^{k}\left(\mathbb{C}^{n}\right)$ can be integrated. Moreover, combining (3.2) with the definition of the Evans function (2.8) leads to the following readily computable expression for the Evans function

$$
E(\lambda)=\llbracket \overline{\mathbf{V}^{-}(0, \lambda)}, \mathbf{U}^{+}(0, \lambda) \rrbracket_{k} .
$$

There are many other ways of formulating the Evans function, including using only solutions of the adjoint (cf. Benzoni-Gavage, Serre \& Zumbrun [9]). The form of the Evans function (3.4) is called a "mixed" Evans function in [9], although it is derived there without using the Hodge star operator.

For the numerical implementation, we will need a basis for $\bigwedge^{k}\left(\mathbb{C}^{n}\right)$, and the above construction assures that any basis will do. Therefore there is no loss of generality is assuming that the bases chosen are the standard ones.

Here we will restrict attention to the case $k=2$ and $n=5$ which is the most interesting case for the fifth-order $\mathrm{KdV}$; see [5] for the details for general $k, n$.

Starting with the standard basis for $\mathbb{C}^{5}$, and volume form $\mathcal{V}=\mathbf{e}_{1} \wedge \cdots \wedge \mathbf{e}_{5}$, let $\mathbf{a}_{1}, \ldots, \mathbf{a}_{10}$ be the induced orthonormal basis on $\bigwedge^{2}\left(\mathbb{C}^{5}\right)$. Using a standard lexical ordering, this basis can be taken to be

$$
\begin{aligned}
& \mathbf{a}_{1}=\mathbf{e}_{1} \wedge \mathbf{e}_{2}, \quad \mathbf{a}_{2}=\mathbf{e}_{1} \wedge \mathbf{e}_{3}, \quad \mathbf{a}_{3}=\mathbf{e}_{1} \wedge \mathbf{e}_{4}, \quad \mathbf{a}_{4}=\mathbf{e}_{1} \wedge \mathbf{e}_{5}, \quad \mathbf{a}_{5}=\mathbf{e}_{2} \wedge \mathbf{e}_{3}, \\
& \mathbf{a}_{6}=\mathbf{e}_{2} \wedge \mathbf{e}_{4}, \quad \mathbf{a}_{7}=\mathbf{e}_{2} \wedge \mathbf{e}_{5}, \quad \mathbf{a}_{8}=\mathbf{e}_{3} \wedge \mathbf{e}_{4}, \quad \mathbf{a}_{9}=\mathbf{e}_{3} \wedge \mathbf{e}_{5}, \quad \mathbf{a}_{10}=\mathbf{e}_{4} \wedge \mathbf{e}_{5} .
\end{aligned}
$$


Any $\mathbf{U} \in \bigwedge^{2}\left(\mathbb{C}^{5}\right)$ can be expressed as $\mathbf{U}=\sum_{j=1}^{10} U_{j} \mathbf{a}_{j}$. Since the basis elements $\mathbf{a}_{i}$ are orthogonal and the inner product $\llbracket \cdot, \cdot \rrbracket_{2}$ on $\bigwedge^{2}\left(\mathbb{C}^{5}\right)$ is equivalent to the inner product $\langle\cdot, \cdot\rangle_{10}$ on $\mathbb{C}^{10}$, the expression (3.4) for the Evans function can be expressed in the equivalent form

$$
E(\lambda)=\left\langle\overline{\mathbf{V}^{-}(0, \lambda)}, \mathbf{U}^{+}(0, \lambda)\right\rangle_{10} .
$$

The matrix $\mathbf{A}^{(2)}: \bigwedge^{2}\left(\mathbb{C}^{5}\right) \rightarrow \bigwedge^{2}\left(\mathbb{C}^{5}\right)$ can be associated with a complex $10 \times 10$ matrix with entries

$$
\left\{\mathbf{A}^{(2)}\right\}_{i, j}=\llbracket \mathbf{a}_{i}, \mathbf{A}^{(2)} \mathbf{a}_{j} \rrbracket_{2}, \quad i, j=1, \ldots, 10,
$$

where, for any decomposable $\mathbf{x}=\mathbf{x}_{1} \wedge \mathbf{x}_{2} \in \bigwedge^{2}\left(\mathbb{C}^{5}\right), \mathbf{A}^{(2)} \mathbf{x}:=\mathbf{A} \mathbf{x}_{1} \wedge \mathbf{x}_{2}+\mathbf{x}_{1} \wedge \mathbf{A} \mathbf{x}_{2}$. Let $\mathbf{A}$ be an arbitrary $5 \times 5$ matrix with complex entries,

$$
\mathbf{A}=\left(\begin{array}{ccccc}
a_{11} & a_{12} & a_{13} & a_{14} & a_{15} \\
a_{21} & a_{22} & a_{23} & a_{24} & a_{25} \\
a_{31} & a_{32} & a_{33} & a_{34} & a_{35} \\
a_{41} & a_{42} & a_{43} & a_{44} & a_{45} \\
a_{51} & a_{52} & a_{53} & a_{54} & a_{55}
\end{array}\right)
$$

then, with respect to the basis (3.5), $\mathbf{A}^{(2)}$ takes the explicit form

$\left[\begin{array}{cccccccccc}a_{11}+a_{22} & a_{23} & a_{24} & a_{25} & -a_{13} & -a_{14} & -a_{15} & 0 & 0 & 0 \\ a_{32} & a_{11}+a_{33} & a_{34} & a_{35} & a_{12} & 0 & 0 & -a_{14} & -a_{15} & 0 \\ a_{42} & a_{43} & a_{11}+a_{44} & a_{45} & 0 & a_{12} & 0 & a_{13} & 0 & -a_{15} \\ a_{52} & a_{53} & a_{54} & a_{11}+a_{55} & 0 & 0 & a_{12} & 0 & a_{13} & a_{14} \\ -a_{31} & a_{21} & 0 & 0 & a_{22}+a_{33} & a_{34} & a_{35} & -a_{24} & -a_{25} & 0 \\ -a_{41} & 0 & a_{21} & 0 & a_{43} & a_{22}+a_{44} & a_{45} & a_{23} & 0 & -a_{25} \\ -a_{51} & 0 & 0 & a_{21} & a_{53} & a_{54} & a_{22}+a_{55} & 0 & a_{23} & a_{24} \\ 0 & -a_{41} & a_{31} & 0 & -a_{42} & a_{32} & 0 & a_{33}+a_{44} & a_{45} & -a_{35} \\ 0 & -a_{51} & 0 & a_{31} & -a_{52} & 0 & a_{32} & a_{54} & a_{33}+a_{55} & a_{34} \\ 0 & 0 & -a_{51} & a_{41} & 0 & -a_{52} & a_{42} & -a_{53} & a_{43} & a_{44}+a_{55}\end{array}\right]$

Applying this algorithm to the linearized system associated with the 5th-order KdV equation 
leads to the induced system,

$$
\mathbf{A}^{(2)}(x, \lambda)=\left(\begin{array}{cccccccccc}
0 & 1 & 0 & 0 & 0 & 0 & 0 & 0 & 0 & 0 \\
0 & 0 & 1 & 0 & 1 & 0 & 0 & 0 & 0 & 0 \\
-\frac{f_{2}(x)}{\beta} & \frac{f_{3}(x)-\alpha}{\beta} & 0 & 1 & 0 & 1 & 0 & 0 & 0 & 0 \\
0 & 0 & 0 & 0 & 0 & 0 & 1 & 0 & 0 & 0 \\
0 & 0 & 0 & 0 & 0 & 1 & 0 & 0 & 0 & 0 \\
-\frac{c+f_{1}(x)}{\beta} & 0 & 0 & 0 & \frac{f_{3}(x)-\alpha}{\beta} & 0 & 1 & 1 & 0 & 0 \\
\frac{\lambda}{\beta} & 0 & 0 & 0 & 0 & 0 & 0 & 0 & 1 & 0 \\
0 & -\frac{c+f_{1}}{\beta} & 0 & 0 & -\frac{f_{2}(x)}{\beta} & 0 & 0 & 0 & 1 & 0 \\
0 & \frac{\lambda}{\beta} & 0 & 0 & 0 & 0 & 0 & 0 & 0 & 1 \\
0 & 0 & \frac{\lambda}{\beta} & \frac{c+f_{1}(x)}{\beta} & 0 & 0 & \frac{f_{2}(x)}{\beta} & 0 & \frac{f_{3}(x)-\alpha}{\beta} & 0
\end{array}\right)
$$

Let $\left\{\mathbf{b}_{1}, \ldots, \mathbf{b}_{d}\right\}$ be an orthogonal basis for $\bigwedge^{3}\left(\mathbb{C}^{5}\right)$. The action of Hodge star is defined by its action on basis vectors $\llbracket \overline{\star \mathbf{b}_{j}}, \mathbf{a}_{i} \rrbracket_{k} \mathcal{V}=\mathbf{b}_{j} \wedge \mathbf{a}_{i}$, for $i, j=1, \ldots d$.

Using a standard lexical ordering, a basis for $\bigwedge^{3}\left(\mathbb{C}^{5}\right)$ can be taken to be

$$
\begin{array}{rlll}
\mathbf{b}_{1}=\mathbf{e}_{1} \wedge \mathbf{e}_{2} \wedge \mathbf{e}_{3}, & \mathbf{b}_{2}=\mathbf{e}_{1} \wedge \mathbf{e}_{2} \wedge \mathbf{e}_{4}, & \mathbf{b}_{3}=\mathbf{e}_{1} \wedge \mathbf{e}_{2} \wedge \mathbf{e}_{5} \\
\mathbf{b}_{4}=\mathbf{e}_{1} \wedge \mathbf{e}_{3} \wedge \mathbf{e}_{4}, & \mathbf{b}_{5}=\mathbf{e}_{1} \wedge \mathbf{e}_{3} \wedge \mathbf{e}_{5}, & \mathbf{b}_{6}=\mathbf{e}_{1} \wedge \mathbf{e}_{4} \wedge \mathbf{e}_{5} \\
\mathbf{b}_{7}=\mathbf{e}_{2} \wedge \mathbf{e}_{3} \wedge \mathbf{e}_{4}, & \mathbf{b}_{8}=\mathbf{e}_{2} \wedge \mathbf{e}_{3} \wedge \mathbf{e}_{5}, & \mathbf{b}_{9}=\mathbf{e}_{2} \wedge \mathbf{e}_{4} \wedge \mathbf{e}_{5} \\
\mathbf{b}_{10}=\mathbf{e}_{3} \wedge \mathbf{e}_{4} \wedge \mathbf{e}_{5} . & &
\end{array}
$$

A matrix representation for the star operator, denoted by $S \in \mathbb{R}^{10 \times 10}$, is then defined by

$$
\star \mathbf{b}_{j}=\sum_{l=1}^{10} S_{j l} \mathbf{a}_{l}, \quad j=1, \ldots, 10, \quad \text { hence } \overline{S_{j i}} \mathcal{V}=\mathbf{b}_{j} \wedge \mathbf{a}_{i}
$$

A straightforward calculation, with the above bases, leads to

$$
\mathbf{S}=\left[\begin{array}{cc}
\mathbf{0} & \mathbf{S}_{1} \\
\mathbf{S}_{1} & \mathbf{0}
\end{array}\right], \quad \mathbf{S}_{1}=\left(\begin{array}{rrrrr}
0 & 0 & 0 & 0 & 1 \\
0 & 0 & 0 & -1 & 0 \\
0 & 0 & 1 & 0 & 0 \\
0 & -1 & 0 & 0 & 0 \\
1 & 0 & 0 & 0 & 0
\end{array}\right)
$$

Note that $\mathbf{S}$ is a symmetric, isometric involution.

Finally, we note another expression for the Evans function which is useful for numerics. Let $\mathbf{U}=\sum_{j=1}^{10} U_{j} \mathbf{a}_{j}$ and $\mathbf{W}=\sum_{j=1}^{10} W_{j} \mathbf{b}_{j}$ then

$$
\mathbf{W} \wedge \mathbf{U}=\llbracket \star \mathbf{W}, \mathbf{U} \rrbracket_{k} \mathcal{V}=\sum_{i, j=1}^{10} S_{j i} W_{j} U_{i}=\langle\overline{\mathbf{W}}, \mathbf{S U}\rangle_{10},
$$

for any $\mathbf{U} \in \bigwedge^{2}\left(\mathbb{C}^{5}\right)$ and $\mathbf{W} \in \bigwedge^{3}\left(\mathbb{C}^{5}\right)$. Hence (3.13) gives a readily computable expression for the original definition (2.8) of the Evans function: $E(\lambda)=\mathrm{e}^{-\int_{0}^{x} \tau(s, \lambda) d s}\left\langle\overline{\mathbf{U}^{-}}, \mathbf{S U}^{+}\right\rangle_{10}$. By letting $\mathbf{V}^{-}(x, \lambda)=\mathrm{e}^{-\int_{0}^{x} \tau(s, \lambda) d s} \mathbf{S} \overline{\mathbf{U}^{-}(x, \lambda)}$ this expression is easily seen to be equivalent to (3.6). 


\section{A shooting algorithm on $\bigwedge^{2}\left(\mathbb{C}^{5}\right)$ and $\bigwedge^{3}\left(\mathbb{C}^{5}\right)$}

The first step in computing the Evans function will be to integrate

$$
\frac{d}{d x} \mathbf{U}^{+}=\mathbf{A}^{(2)}(x, \lambda) \mathbf{U}^{+}, \quad \mathbf{U}^{+} \in \bigwedge^{2}\left(\mathbb{C}^{5}\right)
$$

from $x=L_{\infty}$ to $x=0$ with $\mathbf{A}^{(2)}(x, \lambda)$ as given in (3.9).

Then there are two potential strategies to continue. The first strategy is to integrate the induced system on $\bigwedge^{3}\left(\mathbb{C}^{5}\right)=\bigwedge^{(5-2)}\left(\mathbb{C}^{5}\right)$ :

$$
\frac{d}{d x} \mathbf{U}^{-}=\mathbf{A}^{(3)}(x, \lambda) \mathbf{U}^{-}, \quad \mathbf{U}^{-} \in \bigwedge^{3}\left(\mathbb{C}^{5}\right)
$$

from $x=-L_{\infty}$ to $x=0$ and use the Hodge star operator to match at $x=0$ (see (3.13)),

$$
E(\lambda)=\mathrm{e}^{-\int_{0}^{x} \tau(s, \lambda) d s} \mathbf{U}^{-}(0, \lambda) \wedge \mathbf{U}^{+}(0, \lambda)=\mathrm{e}^{-\int_{0}^{x} \tau(s, \lambda) d s}\left\langle\overline{\mathbf{U}^{-}(0, \lambda)}, \mathbf{S U}^{+}(0, \lambda)\right\rangle_{10},
$$

where the inner product $\langle\cdot, \cdot\rangle_{10}$ is a standard Hermitian inner product on $\mathbb{C}^{10}$. Because conjugation is done twice on $\mathbf{U}^{-}(0, \lambda)$ in $(4.3)$, it remains an analytic function of $\lambda$.

The only negative feature of this construction is that both of the induced systems $\mathbf{A}^{(2)}(x, \lambda)$ and $\mathbf{A}^{(3)}(x, \lambda)$ have to be constructed, although the construction of $\mathbf{A}^{(3)}(x, \lambda)$ can be simplified using $\mathbf{A}^{(3)}=-\mathbf{S}\left[\mathbf{A}^{(2)}\right]^{T} \mathbf{S}$. This relation is proved in $[5,12]$ and follows from the definition of the Hodge star operator.

The second strategy, which is used in the numerics presented in $\S 8$, is to follow the integration of (4.1) with the integration of the complex conjugate equation (3.3)

$$
\frac{d}{d x} \mathbf{V}^{-}=-\left[\mathbf{A}^{(2)}(x, \lambda)\right]^{T} \mathbf{V}^{-}, \quad \mathbf{V}^{-} \in \bigwedge^{2}\left(\mathbb{C}^{5}\right),
$$

from $x=-L_{\infty}$ to $x=0$ and use the matching expression

$$
E(\lambda)=\left\langle\overline{\mathbf{V}^{-}(0, \lambda)}, \mathbf{U}^{+}(0, \lambda)\right\rangle_{10},
$$

deduced from (3.6). In the second strategy, the Hodge star operator is implicit but not explicit in the computation of $E(\lambda)$. This expression is analytic if the construction of the AlExander, GARDNER \& JONES [3] form of the Evans function is analytic, and this is again a consequence of Hodge duality: the Hodge star operator maps holomorphic functions to anti-holomorphic functions (i.e. holomorphic functions of $\bar{\lambda}$ ), and when combined with the inner product results in an analytic inner product (see equation (3.2)). Combining this obervation with the definition of $\mathbf{V}^{-}(x, \lambda)$ confirms that the above expression is analytic. (Holomorphic and analytic are used interchangably here.)

\section{$5 \quad$ Initial conditions at $\pm L_{\infty}$ for the shooting algorithm}

Since the induced matrix $\mathbf{A}^{(k)}(x, \lambda)$ inherits the differentiability and analyticity of $\mathbf{A}(x, \lambda)$, the limiting matrices will exist,

$$
\mathbf{A}_{\infty}^{(k)}(\lambda)=\lim _{x \rightarrow \pm \infty} \mathbf{A}^{(k)}(x, \lambda) .
$$

The set of eigenvalues of the matrix $\mathbf{A}_{\infty}^{(k)}(\lambda)$ consists of all possible $k$-fold sums of the eigenvalues of $\mathbf{A}_{\infty}(\lambda)$ (this is an exercise in multi-linear algebra, see MARcus [42]). Therefore, there is an eigenvalue of $\mathbf{A}_{\infty}^{(k)}(\lambda)$, denoted by $\sigma_{+}(\lambda)$, which is the sum of the $k$ eigenvalues of $\mathbf{A}_{\infty}(\lambda)$ with 
negative real part. Moreover this eigenvalue is simple, an analytic function of $\lambda$ and has real part strictly less than any other eigenvalue of $\mathbf{A}_{\infty}^{(k)}(\lambda)$.

Similarly, there is an eigenvalue of $\mathbf{A}_{\infty}^{(n-k)}(\lambda)$, denoted by $\sigma_{-}(\lambda)$, which is the sum of the $(n-k)$ eigenvalues of $\mathbf{A}_{\infty}(\lambda)$ with non-negative real part, and $\sigma_{-}(\lambda)$ is simple, an analytic function of $\lambda$, and has real part strictly greater than any other eigenvalue of $\mathbf{A}_{\infty}^{(n-k)}(\lambda)$.

Let $\zeta^{ \pm}(\lambda)$ be the eigenvectors associated with $\sigma_{ \pm}(\lambda)$, defined by

$$
\mathbf{A}_{\infty}^{(k)}(\lambda) \zeta^{+}(\lambda)=\sigma_{+}(\lambda) \zeta^{+}(\lambda) \quad \text { and } \quad \mathbf{A}_{\infty}^{(n-k)}(\lambda) \zeta^{-}(\lambda)=\sigma_{-}(\lambda) \zeta^{-}(\lambda) .
$$

These vectors can always be constructed in an analytic way (see below for further aspects of the numerical analytic continuation of these vectors along paths in the complex plane).

The vector $\zeta^{+}(\lambda)$ provides the starting vector at $x=L_{\infty}$ for both the first and second strategies presented in $\S 4$. When the first strategy is used, the vector $\zeta^{-}(\lambda)$ provides the starting vector at $x=-L_{\infty}$ for the system (4.2). When the second strategy is used, we use the fact that $-\overline{\sigma_{+}(\lambda)}$ is an eigenvalue of $-\left[\mathbf{A}_{\infty}^{(k)}\right]^{*}$, and it has real part strictly greater than every other eigenvalue of $-\left[\mathbf{A}_{\infty}^{(k)}\right]^{*}$. Its eigenvector, denoted $\eta^{-}(\lambda)$, and satisfying,

$$
\left[\mathbf{A}_{\infty}^{(k)}(\lambda)\right]^{*} \eta^{-}(\lambda)=\overline{\sigma_{+}(\lambda)} \eta^{-}(\lambda) .
$$

is used as the starting vector. In this case $\eta^{-}(\lambda)$ is normalized so that

$$
\left\langle\eta^{-}(\lambda), \zeta^{+}(\lambda)\right\rangle_{d}=1 .
$$

This may appear to be the natural normalization of an eigenvector with its adjoint eigenvector, but it can also be derived from the geometric normalization, $\zeta^{-} \wedge \zeta^{+}=\mathcal{V}$,

$$
\mathcal{V}=\zeta^{-}(\lambda) \wedge \zeta^{+}(\lambda)=\llbracket \star \zeta^{-}, \zeta^{+} \rrbracket_{k} \mathcal{V}=\llbracket \eta^{-}, \zeta^{+} \rrbracket_{k} \mathcal{V} \quad \Leftrightarrow \quad \llbracket \eta^{-}, \zeta^{+} \rrbracket_{k}=1
$$

since $\operatorname{Ker}\left[\mathbf{A}_{\infty}^{(k)}(\lambda)^{*}-\overline{\sigma_{+}(\lambda)} \mathbf{I}\right]=\operatorname{span}\left\{\star \zeta^{-}(\lambda)\right\}=\operatorname{span}\left\{\eta^{-}\right\}$. This expression when combined with the comments above equation (3.6) recovers (5.7).

When $n \geq 5$ the eigenvalues of $\mathbf{A}_{\infty}(\lambda)$ can not in general be computed analytically, and therefore a numerical scheme is needed which for each $\lambda \in \Lambda$, (a) finds the eigenvalue $\sigma(\lambda)$ of $\mathbf{A}_{\infty}^{(2)}(\lambda)$ of largest negative real part, (b) computes right, $\zeta^{+}(\lambda)$, and left $\eta^{-}(\lambda)$ eigenvectors, and (c) as $\lambda$ is varied ensures that the eigenvalues and eigenvectors vary analytically. It is the latter point (c) which requires special attention.

For the case of the linearization about the fifth-order $\mathrm{KdV}$, the characteristic polynomial is quintic, and its roots can not be determined analytically except for special values of $\lambda$. However, the induced matrix at infinity, $\mathbf{A}_{\infty}^{(2)}(\lambda)$, has a very sparse structure (see (3.9)) and so given the eigenvalue, the eigenvectors $\zeta^{+}(\lambda)$ and $\eta^{-}(\lambda)$ can be computed explicitly and varied analytically. However, in general for other systems on dimension $n \geq 5$ this will not be possible and an algorithm is needed to numerically analytically continue eigenvectors.

\subsection{Analytic $\lambda$-paths of initial conditions at $\pm L_{\infty}$}

In general, if $\zeta^{ \pm}(\lambda)$ are constructed, numerically, at two distinct neighboring points it is not immediate - and indeed unlikely - that one is the analytic continuation of the other. In this section we present a new robust algorithm for analytically continuing the starting vectors. Indeed, the algorithm is quite general and provides a method for numerical analytic continuation of the left and right eigenvectors of any analytic matrix with a simple eigenvalue, and it will be presented in this generality. 
Let $\Lambda$ be an open simply-connected subset of the complex $\lambda$-plane. Suppose $\mathbf{A}(\lambda)$ is any $n \times n$ matrix which depends analytically on $\lambda$ for all $\lambda \in \Lambda$. If $\sigma(\lambda)$ is a simple eigenvalue of $\mathbf{A}(\lambda)$ on $\Lambda$, then there exist analytic eigenvectors $\xi(\lambda)$ and $\eta(\lambda)$ satisfying

$$
\mathbf{A}(\lambda) \xi(\lambda)=\sigma(\lambda) \xi(\lambda), \quad \mathbf{A}(\lambda)^{T} \eta(\lambda)=\sigma(\lambda) \eta(\lambda), \quad \eta(\lambda)^{T} \xi(\lambda)=1 .
$$

To be precise, $\overline{\eta(\lambda)}$ is the adjoint eigenvector, then $\langle\overline{\eta(\lambda)}, \zeta(\lambda)\rangle$, where $\langle\cdot, \cdot\rangle$ is a Hermitian inner product, leads to the third equation above. However, to avoid the double conjugation and to emphasize the analyticity, the transpose will be used.

The analyticity of the eigenvectors follows from Kato's Theorem (KATO [36], pp. 99-101). A straightforward numerical implementation of Kato's Theorem would be quite cumbersome numerically. However BRIN \& ZUMBRUN [17] present a numerical implementation of Kato's Theorem. To analytically continue a vector numerically (from say $\lambda=\lambda_{1}$ to $\lambda=\lambda_{2}$ ), they construct a hybrid method where the (nonanalytic) left and right eigenvectors are computed numerically at $\lambda_{1}$ and $\lambda_{2}$, and they are then used to construct an analytic projection, and the vectorfield in Kato's Theorem.

Here we will propose a new numerical formulation for computing analytic eigenvectors rigorously. Indeed, a by-product of the numerical formulation of analytic continuation introduced here is a new proof of Kato's Theorem. The idea is to construct analytic differential equations for $\sigma(\lambda), \xi(\lambda)$ and $\zeta(\lambda)$ in a way which is different from Kato's construction of an ODE. Moreover, the constructed ODEs are straightforward to integrate numerically.

Differentiating (5.8) with respect to $\lambda$ leads to the equations

$$
\begin{aligned}
{[\mathbf{A}(\lambda)-\sigma(\lambda) \mathbf{I}] \xi^{\prime}(\lambda)-\sigma^{\prime}(\lambda) \xi(\lambda) } & =-\mathbf{A}^{\prime}(\lambda) \xi(\lambda) \\
{\left[\mathbf{A}(\lambda)^{T}-\sigma(\lambda) \mathbf{I}\right] \eta^{\prime}(\lambda)-\sigma^{\prime}(\lambda) \eta(\lambda) } & =-\mathbf{A}^{\prime}(\lambda)^{T} \eta(\lambda) \\
\eta(\lambda)^{T} \xi^{\prime}(\lambda)+\xi(\lambda)^{T} \eta^{\prime}(\lambda) & =0 .
\end{aligned}
$$

The third equation will be replaced by the independent conditions

$$
\eta(\lambda)^{T} \xi^{\prime}(\lambda)=0 \quad \text { and } \quad \xi(\lambda)^{T} \eta^{\prime}(\lambda)=0
$$

which result in unique expressions for $\xi^{\prime}(\lambda)$ and $\eta^{\prime}(\lambda)$. Let $\lambda_{0}$ be any point in $\Lambda$ (the starting point), and let $\sigma_{0}$ be the eigenvalue, $\xi_{0}$ the eigenvector and and $\eta_{0}$ the left eigenvector at $\lambda_{0}$ satisfying (5.8). Combining these equations leads to the pair of coupled nonlinear ODEs,

$$
\begin{gathered}
{\left[\begin{array}{cc}
{[\mathbf{A}(\lambda)-\sigma(\lambda) \mathbf{I}]} & -\xi(\lambda) \\
-\eta(\lambda)^{T} & 0
\end{array}\right]\left(\begin{array}{l}
\xi^{\prime}(\lambda) \\
\sigma^{\prime}(\lambda)
\end{array}\right)=\left(\begin{array}{c}
-\mathbf{A}^{\prime}(\lambda) \xi(\lambda) \\
0
\end{array}\right), \quad\left\{\begin{array}{l}
\xi\left(\lambda_{0}\right)=\xi_{0} \\
\sigma\left(\lambda_{0}\right)=\sigma_{0}
\end{array}\right.} \\
{\left[\begin{array}{cc}
{\left[\mathbf{A}(\lambda)^{T}-\sigma(\lambda) \mathbf{I}\right]} & -\eta(\lambda) \\
-\xi(\lambda)^{T} & 0
\end{array}\right]\left(\begin{array}{l}
\eta^{\prime}(\lambda) \\
\sigma^{\prime}(\lambda)
\end{array}\right)=\left(\begin{array}{c}
-\mathbf{A}^{\prime}(\lambda)^{T} \eta(\lambda) \\
0
\end{array}\right), \quad\left\{\begin{array}{l}
\eta\left(\lambda_{0}\right)=\eta_{0} \\
\sigma\left(\lambda_{0}\right)=\sigma_{0}
\end{array}\right.}
\end{gathered}
$$

Lemma 5.1. Let $\Lambda$ be an open simply-connected subset of the complex $\lambda$-plane. Suppose $\sigma(\lambda)$ is a simple eigenvalue for all $\lambda \in \Lambda$. The nonlinear systems (5.9) and (5.10) provide unique analytic expressions for $\xi^{\prime}(\lambda), \eta^{\prime}(\lambda)$ and $\sigma^{\prime}(\lambda)$, and their solution produces analytic functions $\xi(\lambda), \eta(\lambda)$ and $\sigma(\lambda)$ for all $\lambda \in \Lambda$.

Proof. Given a complex ODE of the form $u_{\lambda}=f(u, \lambda)$ with $f$ Lipschitz in $u$ and analytic in $\lambda$ for all $\lambda \in \Lambda$, there is a unique local solution about any point $\lambda_{0} \in \Lambda$ (cf. CodDington \& LEVINSON [20], page 34). We will show below that for all $\lambda \in \Lambda$ the vectorfield is well defined, and so the solution can be continued to all of $\Lambda$. The proof that (5.9) and (5.10) define unique vectorfields for all $\lambda \in \Lambda$ follows from invertibility of the bordered matrices above. 
Consider the following general homogeneous system

$$
(\mathbf{A}-\sigma \mathbf{I}) \mathbf{u}-\xi v=0 \quad \text { and } \quad-\eta^{T} \mathbf{u}=0,
$$

for $(\mathbf{u}, v) \in \mathbb{C}^{n} \times \mathbb{C}$ under the hypothesis that $\sigma$ is a simple eigenvalue of $\mathbf{A}$ with right and left eigenvectors $\xi$ and $\eta$ normalized so that $\eta^{T} \xi=1$. Multiply the first equation by $\eta^{T}$ to conclude that $v=0$. Then the only solution of the first equation is $\mathbf{u}=a \xi$ where $a \in \mathbb{C}$ is arbitrary. Substitution of this result into the second equation requires $a=0$, so the only solution of the homogeneous equation is the trivial solution. This completes the proof of pointwise invertibility of the bordered matrices in (5.9) and (5.10), and analyticity of the inverse is assured by the fact that $\sigma(\lambda)$ is simple for all $\lambda \in \Lambda$.

An explicit expression for the determinant of the inverse of the above bordered matrix can be obtained by using ideas from the linear algebra of bordered matrices (cf. MAGNUS \& NeUdECKER [43]). For example if $\eta^{T} \xi=1$, then the determinant of the bordered matrix in (5.9) equals minus the product of the nonzero eigenvalues of $\mathbf{A}-\sigma \mathbf{I}$. This result can be used to estimate the distance to the edge of the set $\Lambda$.

Solutions of the differential equations (5.9)-(5.10) provide analytic paths of the vectors $\xi(\lambda)$ and $\eta(\lambda)$ through $\Lambda$. Note also that the surface $\eta(\lambda)^{T} \xi(\lambda)-1=0$ is a quadratic strong invariant manifold of (5.9)-(5.10), since

$$
\frac{d}{d \lambda}\left(\eta(\lambda)^{T} \xi(\lambda)-1\right)=0
$$

independent of the value of $\eta(\lambda)^{T} \xi(\lambda)$. (In the numerical analysis literature a constraint $I$, associated with an ODE, is called a weak constraint if $I_{x}=f(I)$ with $f(0)=0$, and is called a strong constraint if $I_{x}=0$, independent of the value of the constraint.)

Numerical integration of (5.9) and (5.10) will provide a path of analytic starting vectors for the shooting algorithm on $\bigwedge^{k}\left(\mathbb{C}^{n}\right)$. Bordered systems such as $(5.9)$ and $(5.10)$ are widely used in bifurcation and path-following algorithms (cf. GovaERTs [29]); however, numerical analytic continuation does not seem to have been previously considered. Indeed, in the numerical analysis literature, the main emphasis for bordered systems is on development of efficient methods for inversion. Such efficiency could be useful for (5.9)-(5.10), but the dimension for the linearized $\mathrm{KdV}$ is small enough where straightforward numerical inversion is satisfactory.

A more important issue associated with the numerical integration of (5.9) and (5.10) is preservation of the constraint $\eta^{T} \xi=1$. The class of Gauss-Legendre Runge-Kutta (GL-RK) methods are natural for this integration because they preserve strong quadratic constraints - of linear or nonlinear ODEs - to machine accuracy. This result is known in the numerical analysis literature as Cooper's Theorem [21]. (It will appear again in the next section where the Grassmannian which is also quadratic - needs to be preserved.)

Use of a GL-RK method to integrate (5.9)-(5.10) will result in preservation of $\eta(\lambda)^{T} \xi(\lambda)=1$ to machine accuracy. The most obvious choice is the implicit midpoint rule which is the unique second-order GL-RK method. For the implicit midpoint rule, a simple proof of Cooper's Theorem, extended to the complex analytic case, can be given.

Lemma 5.2. Suppose the complex analytic vectorfield $u_{\lambda}=\mathbf{f}(\mathbf{u}, \lambda)$ has a strong quadratic constraint $I(\mathbf{u})=\mathbf{u}^{T} \mathbf{Q u}$ for some constant symmetric matrix $\mathbf{Q}$. Then discretization of this ODE using the implicit midpoint rule preserves the constraint to machine accuracy.

Proof. Strong invariance, $I_{\lambda}=0$, implies $2 \mathbf{u}^{T} \mathbf{Q f}(\mathbf{u}, \lambda)=0$ for all $\lambda$. The implicit midpoint rule discretization of the ODE is

$$
\mathbf{u}^{n+1}=\mathbf{u}^{n}+\Delta \lambda_{n} \mathbf{f}^{n+1 / 2}, \quad \text { for all } n \in \mathbb{N}
$$


where $\Delta \lambda_{n}$ is the variable step size and

$$
\mathbf{f}^{n+1 / 2}=\mathbf{f}\left(\frac{\mathbf{u}^{n+1}+\mathbf{u}^{n}}{2}, \lambda_{n+1 / 2}\right) \quad \text { and } \quad \lambda_{n+1 / 2}=\lambda_{n}+\frac{1}{2} \Delta \lambda_{n} .
$$

The discrete version of strong invariance, $2 \mathbf{u}^{T} \mathbf{Q f}(\mathbf{u}, \lambda)=0$, takes the form

$$
\left(\mathbf{u}^{n}+\mathbf{u}^{n+1}\right)^{T} \mathbf{Q} \mathbf{f}^{n+1 / 2}=0 .
$$

Now

$$
\begin{aligned}
I_{n+1}=\left(\mathbf{u}^{n+1}\right)^{T} \mathbf{Q} \mathbf{u}^{n+1} & =\left(\mathbf{u}^{n+1}\right)^{T} \mathbf{Q}\left(\mathbf{u}^{n}+\Delta \lambda_{n} \mathbf{f}^{n+1 / 2}\right) \\
& =\left(\mathbf{u}^{n+1}\right)^{T} \mathbf{Q} \mathbf{u}^{n}+\Delta \lambda_{n}\left(\mathbf{u}^{n+1}\right)^{T} \mathbf{Q} \mathbf{f}^{n+1 / 2} \\
& =\left(\mathbf{u}^{n}\right)^{T} \mathbf{Q} \mathbf{u}^{n+1}-\Delta \lambda_{n}\left(\mathbf{u}^{n}\right)^{T} \mathbf{Q} \mathbf{f}^{n+1 / 2} \\
& =\left(\mathbf{u}^{n}\right)^{T} \mathbf{Q}\left(\mathbf{u}^{n+1}-\Delta \lambda_{n} \mathbf{f}^{n+1 / 2}\right) \\
& =\left(\mathbf{u}^{n}\right)^{T} \mathbf{Q} \mathbf{u}^{n}=I_{n},
\end{aligned}
$$

proving that the constraint $I$ is preserved exactly (i.e. to machine accuracy) by the discretization.

To summarize: an analytic left eigenvector, $\eta(\lambda)$, and an analytic right eigenvector, $\xi(\lambda)$, associated with a simple eigenvalue $\sigma(\lambda)$ of an analytic matrix $\mathbf{A}(\lambda)$, with analytic normalization $\eta(\lambda)^{T} \xi(\lambda)=1$, can be constructed numerically along any path in $\Lambda$ (where $\Lambda$ is the largest set in which both $\mathbf{A}(\lambda)$ is analytic and $\sigma(\lambda)$ is simple) by integrating (5.9) and (5.10) using the implicit midpoint rule (or other GL-RK integrator).

\subsection{Analytic $\lambda$-paths of initial conditions at $\pm L_{\infty}$}

Analyticity of the Evans function can be maintained numerically even in the case where the eigenvectors are not continued analytically, when the normalization

$$
\left\langle\eta^{-}(\lambda), \zeta^{+}(\lambda)\right\rangle_{10}=1,
$$

is used. Suppose $\zeta^{+}(\lambda+\Delta \lambda)$ is computed independently of $\zeta^{+}(\lambda)$, for some $\Delta \lambda \neq 0$. Then

$$
\lim _{\Delta \lambda \rightarrow 0} \zeta^{+}(\lambda+\Delta \lambda)=\alpha(\lambda) \zeta^{+}(\lambda)
$$

with $\alpha(\lambda)$ in general nonzero. Hence $\zeta^{+}(\lambda+\Delta \lambda)$ is not an analytic continuation of $\zeta^{+}(\lambda)$. Similarly if $\eta^{-}(\lambda+\Delta \lambda)$ is computed independently of $\eta^{-}(\lambda)$, for some $\Delta \lambda \neq 0$. Then

$$
\lim _{\Delta \lambda \rightarrow 0} \eta^{-}(\lambda+\Delta \lambda)=\beta(\lambda) \eta^{-}(\lambda)
$$

with $\beta(\lambda)$ in general nonzero. However, imposing the normalization (5.11) for each $\lambda$ forces $\alpha(\lambda) \overline{\beta(\lambda)}=1$. Now express the Evans function in terms of the eigenvectors,

$$
E(\lambda)=\left\langle\overline{\mathbf{V}^{-}(0, \lambda)}, \mathbf{U}^{+}(0, \lambda)\right\rangle_{10}=\left\langle\eta^{-}(\lambda), \Phi\left(L_{\infty}, \lambda\right) \zeta^{+}(\lambda)\right\rangle_{10},
$$

where $\Phi\left(L_{\infty}, \lambda\right)$ is the fundamental solution matrix (which is analytic) taking $\zeta^{+}(\lambda)$ from $x=L_{\infty}$ to $x=-L_{\infty}$. Evaluating the perturbed Evans function on this representation,

$$
\begin{aligned}
\lim _{\Delta \lambda \rightarrow 0} E(\lambda+\Delta \lambda) & =\lim _{\Delta \lambda \rightarrow 0}\left\langle\eta^{-}(\lambda+\Delta \lambda), \Phi\left(L_{\infty}, \lambda+\Delta \lambda\right) \zeta^{+}(\lambda+\Delta \lambda)\right\rangle_{10} \\
& =\alpha(\lambda) \overline{\beta(\lambda)}\left\langle\eta^{-}(\lambda), \Phi\left(L_{\infty}, \lambda\right) \zeta^{+}(\lambda)\right\rangle_{10} \\
& =E(\lambda) .
\end{aligned}
$$

In other words, the non-analyticity of the two eigenvectors conveniently cancels out: two wrongs make a right! Therefore if only the eigenvalues are of interest, this method is satisfactory, but if the eigenfunctions $\mathbf{U}^{ \pm}(x, \lambda)$ are of interest, then the methods of $\S 5.1$ are preferred. 


\section{Intermezzo: the Grassmannian is an invariant manifold}

The starting vectors $\zeta^{ \pm}(\lambda)$ and $\eta^{-}(\lambda)$ are decomposable. A decomposable $k$-form represents a $k$-dimensional subspace of $\mathbb{C}^{n}$. In the present example, $\zeta^{+}(\lambda)$ represents the 2 -dimensional subspace of solutions of $\mathbf{v}_{x}=\mathbf{A}_{\infty}(\lambda) \mathbf{v}$ which decays exponentially as $x \rightarrow+\infty$. It is important for the differential equation to preserve this property: if the starting vector for (4.1) is decomposable, is the solution $\mathbf{U}^{+}(x, \lambda)$ decomposable for all $x$ ? We will prove this to be the case below. Indeed, geometrically, the set of decomposable vectors is the Grassmannian $G_{2}\left(\mathbb{C}^{5}\right)$, and what we will prove is that $G_{2}\left(\mathbb{C}^{5}\right)$ is an invariant manifold of (4.1). A consequence of this observation is that it is misleading to view the system of ODEs on $\Lambda^{2}\left(\mathbb{C}^{5}\right)$ as a linear system, since any solution path is required to remain on a submanifold of the ambient linear space.

Preservation of this invariant manifold by the numerics will be a desirable property of the numerical integrator. Even though the continuous system may preserve the manifold, small numerical errors will lead to drift off the manifold, and the manifold may be repelling in the ambient space. An example to illustrate this is given at the end of this section.

The set of all decomposable 2 -forms is a quadratic submanifold of the projectified ambient space $\mathbb{C}^{10}$. This manifold is the Plücker embedding of the Grassmannian $G_{2}\left(\mathbb{C}^{5}\right)$ (see HARRIS [32]). An explicit expression for this quadric is obtained as follows.

Introduce an orthonormal basis for $\bigwedge^{4}\left(\mathbb{C}^{5}\right)$, for example,

$$
\begin{aligned}
& \mathbf{c}_{1}=-\mathbf{e}_{1} \wedge \mathbf{e}_{2} \wedge \mathbf{e}_{4} \wedge \mathbf{e}_{5}, \quad \mathbf{c}_{2}=-\mathbf{e}_{2} \wedge \mathbf{e}_{3} \wedge \mathbf{e}_{4} \wedge \mathbf{e}_{5}, \quad \mathbf{c}_{3}=\mathbf{e}_{1} \wedge \mathbf{e}_{3} \wedge \mathbf{e}_{4} \wedge \mathbf{e}_{5}, \\
& \mathbf{c}_{4}=\mathbf{e}_{1} \wedge \mathbf{e}_{2} \wedge \mathbf{e}_{3} \wedge \mathbf{e}_{5}, \quad \mathbf{c}_{5}=-\mathbf{e}_{1} \wedge \mathbf{e}_{2} \wedge \mathbf{e}_{3} \wedge \mathbf{e}_{4} .
\end{aligned}
$$

The curious ordering here is chosen to give a neat form to the differential equation satisfied by the quadrics.

An element $\mathbf{U} \in \bigwedge^{2}\left(\mathbb{C}^{5}\right)$ is decomposable if and only if $\mathbf{U} \wedge \mathbf{U}=0$ (cf. HARRIs [32], note that this simple characterization of decomposability does not generalize to $k>2$ ). A straightforward calculation, using the bases for $\bigwedge^{2}\left(\mathbb{C}^{5}\right)$ and $\bigwedge^{3}\left(\mathbb{C}^{5}\right)$ introduced in $\S 3$, leads to

$$
\mathbf{U} \wedge \mathbf{U}=2 \sum_{j=1}^{5} I_{j} \mathbf{c}_{j},
$$

where $I_{1}, \ldots, I_{5}$ are defined by

$$
\begin{aligned}
& I_{1}=-u_{1} u_{9}+u_{2} u_{7}-u_{4} u_{5} \\
& I_{2}=-u_{5} u_{10}+u_{6} u_{9}-u_{7} u_{8} \\
& I_{3}=u_{2} u_{10}-u_{3} u_{9}+u_{4} u_{8} \\
& I_{4}=u_{1} u_{8}-u_{2} u_{6}+u_{3} u_{5} \\
& I_{5}=-u_{1} u_{10}+u_{3} u_{7}-u_{4} u_{6} .
\end{aligned}
$$

These quadrics are not all independent, they satisfy the two relations,

$$
u_{2} I_{3}+u_{3} I_{4}+u_{4} I_{5}=0 \text { and } u_{5} I_{3}+u_{6} I_{4}+u_{7} I_{5}=0 .
$$

The quadric surface defined by $\mathbf{I}=0$, where $\mathbf{I}=\left(I_{1}, \ldots, I_{5}\right) \in \mathbb{R}^{5}$, is the Grassmanian $G_{2}\left(\mathbb{C}^{5}\right)$. The $5 \times 10$ matrix $\nabla_{u} \mathbf{I}$ has rank 3 .

The main result about these invariants here is that when $\mathbf{I}$ is evaluated on a solution of an induced system on $\bigwedge^{2}\left(\mathbb{C}^{5}\right)$ of the form (4.1) it satisfies the remarkable equation

$$
\frac{d}{d x} \mathbf{I}=\tau(x, \lambda) \mathbf{I}-\mathbf{A}(x, \lambda)^{T} \mathbf{I} .
$$


For the case of the 5th-order KdV stability equation, this equation simplifies since $\tau=0$. It is immediate from (6.3) that - mathematically - if $\mathbf{I}=0$ at the starting value, it is preserved by the differential equation on $\bigwedge^{2}\left(\mathbb{C}^{5}\right): G_{2}\left(\mathbb{C}^{5}\right)$ is an invariant manifold of (4.1).

On the other hand, numerically these invariants may not be preserved. The vectorfield $\mathbf{I}_{x}$ is not identically zero, but vanishes in general only when $\mathbf{I}=0$. In the numerical analysis literature, this would be called a weak constraint, and we know of no numerical integrator which will preserve a weak constraint exactly. On the other hand, when $k=2$ and $n=4$, the class of GL-RK methods does preserve the Grassmannian $G_{2}\left(\mathbb{C}^{4}\right)$ to machine accuracy $[2,5]$. Therefore in the numerics reported here, we will use the second-order GL-RK method, and monitor the value of $\|\mathbf{I}(x)\|$ as a measure of the geometric error.

An elementary example, which illustrates the importance of the choice of integrator when an invariant manifold of a linear equation needs to be preserved, is the harmonic oscillator

$$
\mathbf{u}_{x}=\mathbf{A u}, \quad \mathbf{u} \in \mathbb{R}^{2}, \quad \mathbf{A}=\left(\begin{array}{rr}
0 & -1 \\
1 & 0
\end{array}\right)
$$

This equation preserves the invariant manifold $I=\|\mathbf{u}\|^{2}$. Look at three standard discretizations of (6.4)

$$
\begin{aligned}
\mathbf{u}^{n+1}=[\mathbf{I}+\Delta x \mathbf{A}] \mathbf{u}^{n} & \text { (forward Euler) } \\
\mathbf{u}^{n+1}=[\mathbf{I}-\Delta x \mathbf{A}]^{-1} \mathbf{u}^{n} & \text { (backward Euler) } \\
\mathbf{u}^{n+1}=\left[\mathbf{I}-\frac{1}{2} \Delta x \mathbf{A}\right]^{-1}\left[\mathbf{I}+\frac{1}{2} \Delta x \mathbf{A}\right] \mathbf{u}^{n} & \text { (implicit midpoint method) }
\end{aligned}
$$

The effect on the invariant manifold of these discretizations is

$$
\begin{array}{rlrl}
I^{n+1}= & \left(1+\Delta x^{2}\right) I^{n} & & \text { (forward Euler) } \\
I^{n+1}=\left(1+\Delta x^{2}\right)^{-1} I^{n} & & \text { (backward Euler) } \\
I^{n+1}=I^{n} & & \text { (implicit midpoint method). }
\end{array}
$$

Both forward and backward Euler escape from the invariant manifold exponentially, whereas the implicit midpoint rule, which is the unique second-order GL-RK method, preserves the invariant manifold in the discretization exactly (i.e. to machine accuracy). In other words, when either forward or backward Euler are used, the geometric error overwhelms the problem. Higher order explicit methods - such as higher-order explicit RK methods - will not do much better than forward Euler. An example, where different higher-order numerical methods are tested for their ability to remain on or near an invariant manifold in an ambient Euclidean space, is given in $\S 6$ of BRIDGEs \& REICH [15]. General aspects of the numerical analysis of ODEs restricted to invariant submanifolds is given in Ascher, CHIN \& REICH [6].

In the case $k=2$ and $n=4$ it is proved in [5] that level sets $I$ of the Grassmanian $G_{2}\left(\mathbb{C}^{4}\right)$ satisfy $I_{x}=\tau(x, \lambda) I$ where $\tau(x, \lambda)$ is the trace of the matrix $\mathbf{A}(x, \lambda)$ on $\mathbb{C}^{4}$, and when $\tau=0$ any GL-RK method will preserve the Grassmanian $G_{2}\left(\mathbb{C}^{4}\right)$ to machine accuracy: indeed, this is a special case of Cooper's Theorem [21]. However, when an explicit method is used, the Grassmannian is not preserved. For example, using forward Euler,

$$
I^{n+1}=2 \Delta x \tau_{n} I^{n}+\mathcal{O}\left(\Delta x^{2}\right) \quad \text { where } \quad G_{2}\left(\mathbb{C}^{4}\right)=I^{-1}(0),
$$

and the $\mathcal{O}\left(\Delta x^{2}\right)$ term does not vanish in general, even when $\tau=0$.

\subsection{Can the Grassmannian be more attractive ?}

In the present case, the GL-RK method will not preserve the Grassmanian $G_{2}\left(\mathbb{C}^{5}\right)$ exactly. Our numerical results indicate however, that the Grassmanian is preserved to within truncation error 
when the implicit midpoint method is used. This is comforting, considering that it is not clear whether $G_{2}\left(\mathbb{C}^{5}\right)$ is a stable or unstable invariant manifold of the ODE $(4.1)$ on $\bigwedge^{2}\left(\mathbb{C}^{5}\right)$, and the asymptotic behaviour of (6.3) suggests that it is unstable. It is an interesting open problem to develop a numerical method which either preserves $G_{2}\left(\mathbb{C}^{5}\right)$ to machine accuracy, or a method or formulation which converts $G_{2}\left(\mathbb{C}^{5}\right)$ to an attracting invariant manifold. An example of the latter is obtained by adding an appropriately chosen term to (4.1) which vanishes when $\mathbf{I}=\mathbf{0}$ and is dissipative otherwise, for example,

$$
\frac{d}{d x} \mathbf{U}^{+}=\mathbf{A}^{(2)}(x, \lambda) \mathbf{U}^{+}-\gamma(\mathbf{I}) \mathbf{U}^{+},
$$

where $\gamma$ is a scalar-valued function satisfying $\gamma(\mathbf{0})=0$, and chosen so that $\mathbf{I}=0$ is attracting.

An example of this approach is to subtract off the exponential growth rate of the most unstable direction and integrate a scaled ODE. For example, consider the integration of the induced system on $\bigwedge^{k}\left(\mathbb{C}^{n}\right)$ from $x=L_{\infty}$ to $x=0$, and introduce the scaled variable $\widetilde{\mathbf{U}}^{+}(x, \lambda)=$ $\mathrm{e}^{-\sigma_{+}(\lambda) x} \mathbf{U}^{+}(x, \lambda)$. Then $\widetilde{\mathbf{U}}^{+}$satisfies the modified ODE

$$
\tilde{\mathbf{U}}_{x}^{+}=\left[\mathbf{A}^{(2)}(x, \lambda)-\sigma_{+}(\lambda) \mathbf{I}_{d}\right] \widetilde{\mathbf{U}}^{+},\left.\quad \widetilde{\mathbf{U}}^{+}(x, \lambda)\right|_{x=L_{\infty}}=\zeta^{+}(\lambda) .
$$

When $\mathbf{A}^{(2)}(x, \lambda)$ is independent of $x$, the vector $\zeta^{+}(\lambda)$ is then a fixed point: the vector corresponding to the fastest growth rate has "neutralized". Now, evaluating the level sets of the Grassmanian, I, on $\widetilde{\mathbf{U}}^{+}$, instead of on $\mathbf{U}^{+}$, leads to a modified form of equation (6.3)

$$
\frac{d}{d x} \widetilde{\mathbf{I}}=\tau(x, \lambda) \widetilde{\mathbf{I}}-\mathbf{A}(x, \lambda)^{T} \widetilde{\mathbf{I}}-2 \sigma_{+}(\lambda) \widetilde{\mathbf{I}},
$$

When $\mathbf{A}(x, \lambda)$ is constant coefficient, then it is clear that the Grassmannian is stable; indeed could be attracting, for in that case $\tau=\sigma_{+}+\sigma_{-}$and the spectrum of the (constant coefficient) matrix on the right-hand side of (6.8) would be in the right-half plane (and therefore stable for integration in the negative $x$-direction). See $\S 8$ for a numerical implementation of this algorithm. A full analysis of this algorithm for the case where $\mathbf{A}(x, \lambda)$ is not constant coefficient case would be of great interest.

\section{Details of the system at infinity for linearized 5th-order $\mathrm{KdV}$}

In this section we present the detailed properties of the eigenvalues of $\mathbf{A}_{\infty}(\lambda)$ for the linearized stability equations associated with the 5 th-order $\mathrm{KdV}$. The characteristic polynomial associated with $\mathbf{A}_{\infty}(\lambda)$ is given in (2.7). At $\lambda=0$, the characteristic polynomial reduces to

$$
\Delta(\mu, 0)=\mu\left(\mu^{4}-\rho_{3} \mu^{2}-\rho_{2} \mu-\rho_{1}\right),
$$

so $\mathbf{A}_{\infty}(0)$ has one zero eigenvalue and the position of the other eigenvalues depends on the parameter values. There are three cases:

- If $\rho_{1}<0$, then there are 2 eigenvalues with positive real part and 2 with negative real part, or all eigenvalues are on the imaginary axis, since the sum of all eigenvalues must be zero and the product of all eigenvalues is positive. However, the case with all eigenvalues on the imaginary axis can only occur if $\rho_{2}=0$ and this case will be considered later.

- If $\rho_{1}>0$ and $\rho_{2}<0$, then there is 1 positive eigenvalue and 3 eigenvalues with negative real part.

- If $\rho_{1}>0$ and $\rho_{2}>0$, then there is 1 negative eigenvalue and 3 eigenvalues with positive real part. 
To verify the second and third observations, we first note that the product of all non-zero eigenvalues must be negative, hence there must be a 1-3 split. Denote the non-zero eigenvalues by $\mu_{1}, \mu_{2}, \mu_{3}$, and $\mu_{4}$, where the sign of $\mu_{1}$ is opposite that of the other 3 eigenvalues and $\mu_{3}=\overline{\mu_{4}}$, if there are eigenvalues with nonzero imaginary part. Since the sum of the eigenvalues is zero, we get that $-\mu_{1}=\mu_{2}+\mu_{3}+\mu_{4}$ and hence

$$
\begin{aligned}
\rho_{2} & =-\left(\mu_{1} \mu_{2} \mu_{3}+\mu_{1} \mu_{2} \mu_{4}+\mu_{1} \mu_{3} \mu_{4}+\mu_{2} \mu_{3} \mu_{4}\right) \\
& =\left(\mu_{2}+\mu_{3}+\mu_{4}\right)\left(\mu_{2}\left(\mu_{3}+\mu_{4}\right)+\mu_{3} \mu_{4}\right)-\mu_{2} \mu_{3} \mu_{4} \\
& =\mu_{2}\left(\mu_{3}+\mu_{4}\right)^{2}+\mu_{2}^{2}\left(\mu_{3}+\mu_{4}\right)+\mu_{3} \mu_{4}\left(\mu_{3}+\mu_{4}\right) .
\end{aligned}
$$

Note that $\mu_{3}+\mu_{4}$ and $\mu_{2}$ are always real and have the same sign. Hence $\left(\mu_{3}+\mu_{4}\right)^{2}>0$ and $\mu_{2}^{2}>0$. Also, $\mu_{3} \mu_{4}>0$. Hence $\mu_{1}$ has the opposite sign of $\rho_{2}$.

Now consider the case $\lambda \neq 0$. For $\lambda$ near 0 , the eigenvalue bifurcating from zero is given by $\mu=\frac{1}{\rho_{1}} \tilde{\lambda}+\mathcal{O}\left(\lambda^{2}\right)$, where $\tilde{\lambda}=\lambda / \beta$.

To determine whether any eigenvalues can cross the imaginary axis for $\lambda$ real and positive, we substitute $\mu=\mathrm{i} \nu$ in the characteristic polynomial, this gives

$$
\Delta(\mathrm{i} \mu, \lambda)=\mathrm{i} \nu\left(\nu^{4}+\rho_{3} \nu^{2}-\rho_{1}\right)+\rho_{2} \nu^{2}+\tilde{\lambda} .
$$

This equation has real solutions $\nu$ if and only if

$$
\left.\begin{array}{l}
0=\rho_{2} \nu^{2}+\tilde{\lambda} \\
0=\nu^{4}+\rho_{3} \nu^{2}-\rho_{1},
\end{array}\right\} \text { hence } \quad\left\{\begin{aligned}
\tilde{\lambda} & =-\rho_{2} \nu^{2} \\
2 \nu^{2} & =-\rho_{3} \pm \sqrt{\rho_{3}^{2}+4 \rho_{1}} .
\end{aligned}\right.
$$

In other words, no real solutions $\nu$ are possible for $\lambda>0$ if $\beta \rho_{2} \geq 0$ or if $4 \rho_{1}<-\rho_{3}^{2}$, or if $\rho_{1}<0$ and $\rho_{3}>0$. In the other cases, real solutions will occur and the split in the eigenvalues will not be constant for all real positive values of $\lambda$.

In this paper, we will restrict attention to the following cases, all of which lead to a 2-3 splitting: when $\beta<0$ there are 2 eigenvalues with negative real part and 3 with positive, and when $\beta>0$ there are 3 eigenvalues with negative real part and 2 with positive.

- $f_{2}^{\infty}>0$ and $\beta\left(f_{1}^{\infty}+c\right)<0$;

- $f_{2}^{\infty}<0$ and $4 \beta\left(f_{1}^{\infty}+c\right)<\left(\alpha-f_{3}^{\infty}\right)^{2}$;

- $f_{2}^{\infty}<0$ and $\beta\left(f_{1}^{\infty}+c\right)<0$ and $\beta\left(f_{3}^{\infty}-\alpha\right)>0$;

Also, $f_{2}^{\infty}>0$ and $\beta\left(f_{1}^{\infty}+c\right)>0$ leads to 1 eigenvalue with negative real part and 4 eigenvalues with positive real part, if $\beta>0$, and the other way around of $\beta<0$. In the other cases there will be some real positive values of $\lambda$ for which the imaginary axis will be crossed and hence the split in the eigenvalues is not constant.

Next we consider the case $f_{2}^{\infty}=0$. At $\lambda=0$, we have $\Delta(\mu, 0)=\mu\left(\mu^{4}-\rho_{3} \mu^{2}-\rho_{1}\right)$. So $\mathbf{A}_{\infty}(0)$ has one zero eigenvalue and the other eigenvalues satisfy

$$
\mu^{2}=\frac{\rho_{3}}{2} \pm \frac{1}{2} \sqrt{\rho_{3}^{2}+4 \rho_{1}} .
$$

See Figure 1 for a sketch of the position of the eigenvalues as function of $\rho_{1}$ and $\rho_{3}$.

To analyse the behaviour near $\lambda=0$, we write $\mu=\mu_{0}+\mu_{1} \tilde{\lambda}+\mathcal{O}\left(\lambda^{2}\right)$, where $\mu_{0}=0$ or satisfies (7.1). In the last case $\mu_{1}$ satisfies

$$
\left(5 \mu_{0}^{4}-3 \rho_{3} \mu_{0}^{2}-\rho_{1}\right) \mu_{1}+1=0,
$$




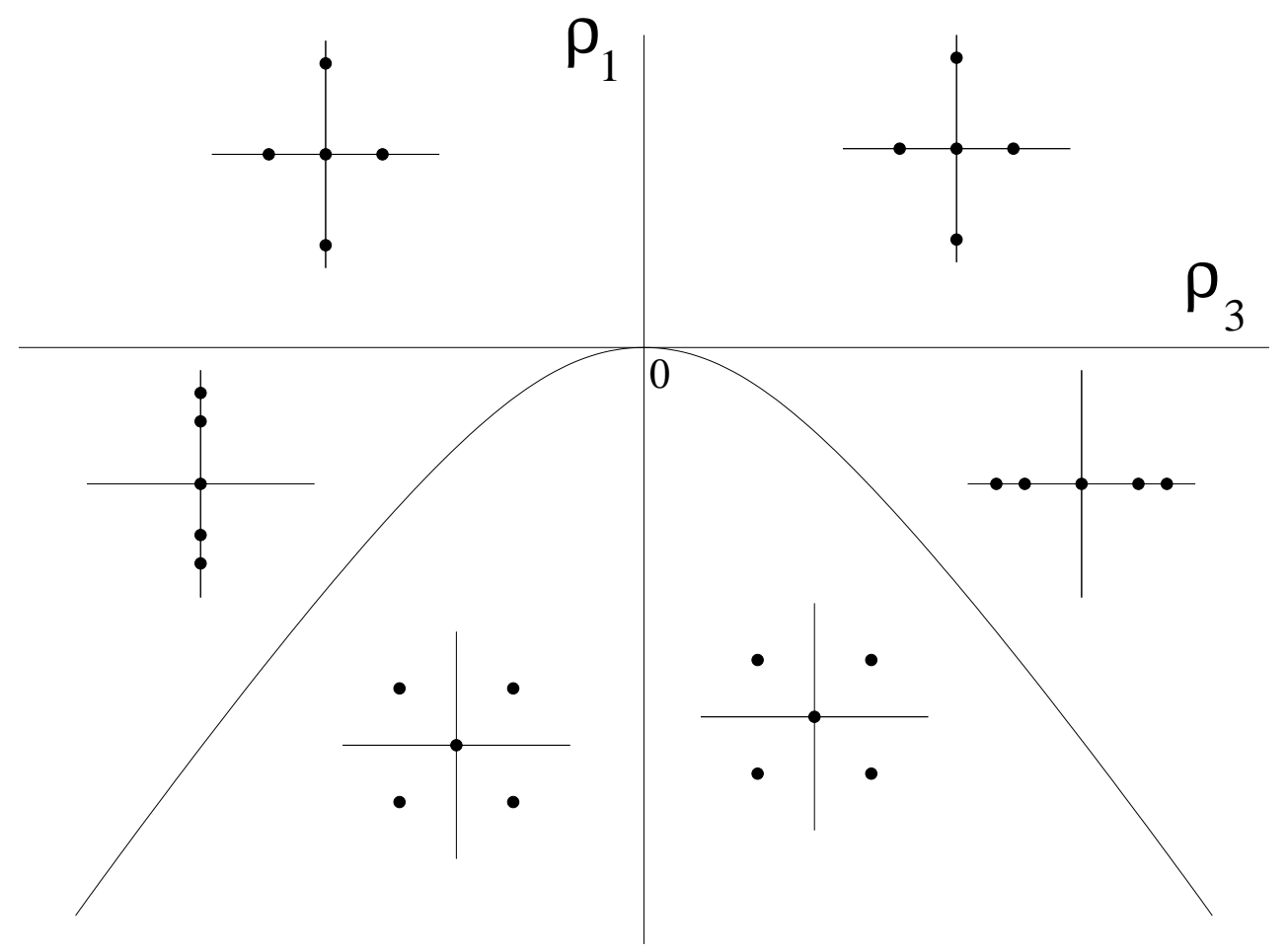

Figure 1: Sketch of the position of the eigenvalues $\mu$ at $\lambda=0$ when $\rho_{2}=f_{2}^{\infty}=0$ as a function of $\rho_{1}$ and $\rho_{3}$. The parabolic curve represents the relation $4 \rho_{1}+\rho_{3}^{2}=0$.

hence

$$
\mu_{1}=-\frac{1}{5 \mu_{0}^{4}-3 \rho_{3} \mu_{0}^{2}-\rho_{1}}=-\frac{1}{2 \rho_{3} \mu_{0}^{2}+4 \rho_{1}}=-\frac{1}{\sqrt{\rho_{3}^{2}+4 \rho_{1}}\left(\sqrt{\rho_{3}^{2}+4 \rho_{1}} \pm \rho_{3}\right)} .
$$

So we have the following cases

- If $\rho_{1}>0$, then at $\lambda=0$, there are 3 eigenvalues on the imaginary axis, one zero, one on the positive imaginary axis and one on the negative imaginary axis. When $\lambda$ is perturbed away from zero, the zero eigenvalue moves to the right, if $\beta>0$, to the left if $\beta<0$, and the nonzero eigenvalues on the imaginary axis move to the left. Hence we get a 3-2 split: 3 eigenvalues with negative real part and 2 eigenvalues with positive real part, if $\beta>0$ and the other way around, $2-3$ split if $\beta<0$.

- If $4 \rho_{1}<-\rho_{3}^{2}$, then at $\lambda=0$, there are 2 eigenvalues with negative real part, 2 eigenvalues with positive real part and one zero eigenvalue. When $\lambda$ is perturbed away from zero, the zero eigenvalue moves to the right, if $\beta>0$ (to the left if $\beta<0$ ). Hence we get a $3-2$ split: 3 eigenvalues with negative real part and 2 eigenvalues with positive real part, if $\beta>0$ (and the other way around if $\beta<0$ ).

- If $-\rho_{3}^{2}<4 \rho_{1}<0$ and $\rho_{3}>0$, then at $\lambda=0$, there are 2 negative eigenvalues, 2 positive eigenvalues and one at zero. Under $\lambda$ perturbation, the zero eigenvalue moves to the right, if $\beta>0$ (to the left if $\beta<0$ ). Hence we get a 3-2 split: 3 eigenvalues with negative real part and 2 eigenvalues with positive real part, if $\beta>0$ (and the other way around when $\beta<0)$.

- If $-\rho_{3}^{2}<4 \rho_{1}<0$ and $\rho_{3}<0$, then at $\lambda=0$, all eigenvalues are on the imaginary axis. Under $\lambda$ perturbation, one pair of eigenvalues moves to the left and one pair of eigenvalues 
moves to the right. The zero eigenvalue moves to the right, if $\beta>0$ (to the left if $\beta<0$ ). Hence we get a 3-2 split: 3 eigenvalues with negative real part and 2 eigenvalues with positive real part, if $\beta>0$ (and the other way around, if $\beta<0$ ).

To summarize, if $f_{2}^{\infty}=0$, there is a $3-2$ split, with 3 eigenvalues with negative real part and 2 eigenvalues with positive real part when $\beta>0$ (and $2-3$ when $\beta<0$ ).

\section{Numerical results for a class of solitary waves}

To demonstrate the numerical framework, we will compute eigenvalues for a class of solitary waves of the 5th-order KdV with polynomial nonlinearity. Consider,

$$
u_{t}+\alpha u_{x x x}+\beta u_{x x x x x}=\partial_{x} f\left(u, u_{x}, u_{x x}\right) \quad \text { with } \quad f\left(u, u_{x}, u_{x x}\right)=K u^{p+1} .
$$

The stability of solitary waves of this equation has been recently considered by KARPMAN [35] and Dey, Khare \& Kumar [23]. Karpman gives two results. The first gives a sufficient condition for stability $\frac{d}{d c} P>0$, where $P$ is the momentum evaluated on the solitary wave. But this condition relies on a conjecture, which has yet to be verified (see the paragraph below equation (39) in [35]). The second condition is independent of the conjecture and also gives a sufficient condition for stability. We will call this result Karpman's condition, because as we will show below, numerical evidence suggests that it may be sharp.

Dey, Khare \& Kumar show that (8.1) has an explicit solitary wave solution of the form

$$
u(x, t)=A^{\frac{1}{p}} \operatorname{sech}^{\frac{4}{p}}(B(x-c t)),
$$

with $c=-\frac{4 \alpha^{2}}{\beta}(p+2)^{2}\left(p^{2}+4 p+8\right)^{-2}$ and

$$
A=\frac{\alpha^{2}}{2 \beta K} \frac{(p+4)(3 p+4)(p+2)}{\left(p^{2}+4 p+8\right)^{2}}, \quad B^{2}=-\frac{\alpha}{4 \beta} \frac{p^{2}}{p^{2}+4 p+8},
$$

with the required conditions $\alpha \beta<0$ and $\beta K>0$. We will call this the DKK solution. They apply Karpman's condition to this wave to show that a sufficient condition for the solitary wave to be stable is precisely when

$$
3 p^{5}+28 p^{4}-608 p^{2}-1664 p-1024<0 .
$$

An approximate value of $p$ where the sign of this polynomial changes is $p_{\text {crit }}=4.84$. There is no indication in Karpman's theory that this value of $p$ might be sharp. Indeed, Dey, Khare \& KUmAR are only able to conclude that the solitary wave is unstable if $p \geq 5$.

Fix the parameters at $\alpha=+1, \beta=-1$ and $K=-1$. The model under consideration is then

$$
u_{t}+(p+1) u^{p} u_{x}+u_{x x x}-u_{x x x x x}=0 .
$$

In this case, the speed of the DKK solution satisfies $0<c<\frac{1}{4}$.

The system at infinity, $\mathbf{A}_{\infty}(\lambda)$, has characteristic polynomial,

$$
\Delta(\mu, \lambda)=\mu^{5}-\mu^{3}+c \mu-\lambda .
$$

When $\lambda=0$ there are four real distinct eigenvalues, two positive and two negative (see Figure 1 with $\rho_{3}>0$ and $\rho_{1}<0$ ), and when $\lambda$ is perturbed away from zero, along the positive real axis, there is a $2-3$ splitting: two eigenvalues with negative real part and three with positive. The dual problem, taking $\alpha=-1, \beta=+1$ and $K=+1$ results in $c<0$ and so the structure of the system at infinity is equivalent, but with a $3-2$ splitting. 
At $x=20$, the amplitude of the basic solitary wave (8.2) is of order $10^{-11}$, and so in all the calculations, $L_{\infty}=20$.

In the first set of calculations, the Evans function is evaluated along the real $\lambda$-axis. The Evans function has the property that $E(\lambda)$ is real when $\lambda$ is real. In this case we find that there is a real unstable eigenvalue when $p$ is large enough. On the other hand, when there are no unstable real eigenvalues, we then use Cauchy's Theorem (Argument Principle) to numerically count eigenvalues in the positive right half plane.

In Figure 2 the computed Evans function for the linearization about (8.2) is shown with $p=4$ and $p=5$. Even for $p=10$, the growth rate of the unstable eigenvalue is still quite small, see Figure 3.

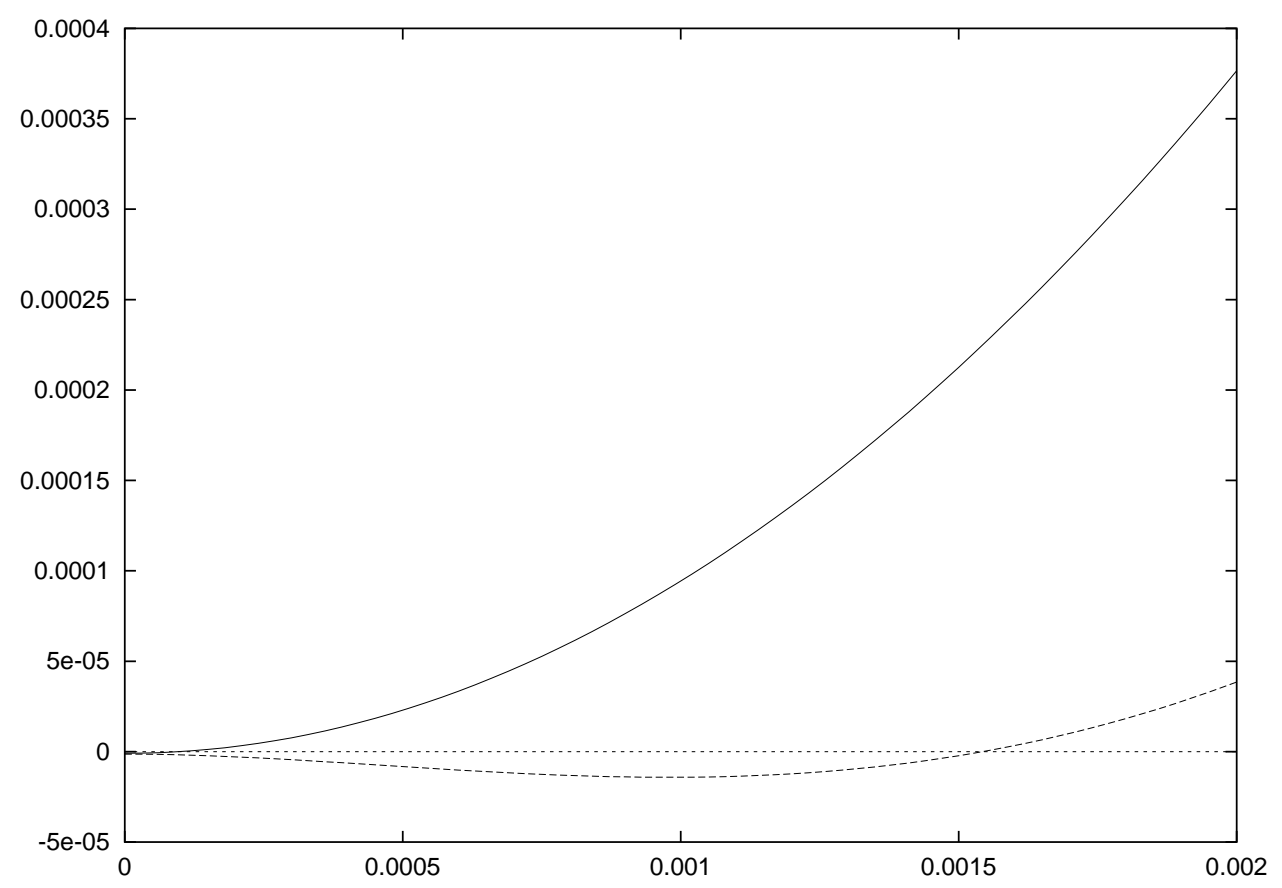

Figure 2: Plot of the Evans function versus $\lambda$ along the real axis, for the linearization about (8.2), with $p=4$ (continuous line) and $p=5$ (dashed line).

It is evident that there is an unstable real eigenvalue for $p \geq 5$, and it is stable for $p \leq 4$. Allowing $p$ to be a real number, there is clearly a stability change for some $p$ between 4 and 5. More refined calculations show that the change occurs at approximately 4.80 , see Figure 4 . We note that the chosen resolution does not result in $E(0)=0$, but $E(0) \approx 10^{-9}$, and that the values near $\lambda=0$ are too small to allow for any precise numerical value of $p_{\text {crit }}$. Nevertheless, we see a trend in our simulations of the change at the second derivative of $E(\lambda)$ near $\lambda=0$. Taking into account the numerical accuracy, the computed value of $p_{\text {crit }}$ provides strong evidence that Karpman's condition may be sharp in this case.

The procedure for the numerical calculations is as follows. As explained in Section 4, it is sufficient to restrict the shooting algorithm to $\Lambda^{2}\left(\mathbb{C}^{5}\right)$. As a starting vector for the shooting algorithm we need to determine the eigenvalues of $\mathbf{A}_{\infty}^{(2)}(\lambda)$ in the far-field. For the integration starting at $x=+L_{\infty}$ the starting vectors for each $\lambda$ are the eigenvectors $\zeta^{+}(\lambda)$ with the largest negative real part; for the integration starting at $x=-L_{\infty}$ the starting vectors are the eigenvectors $\eta^{-}(\lambda)$ with the largest positive real part. They are normalized so that $\left\langle\eta^{-}(\lambda), \zeta^{+}(\lambda)\right\rangle_{10}=1$. 


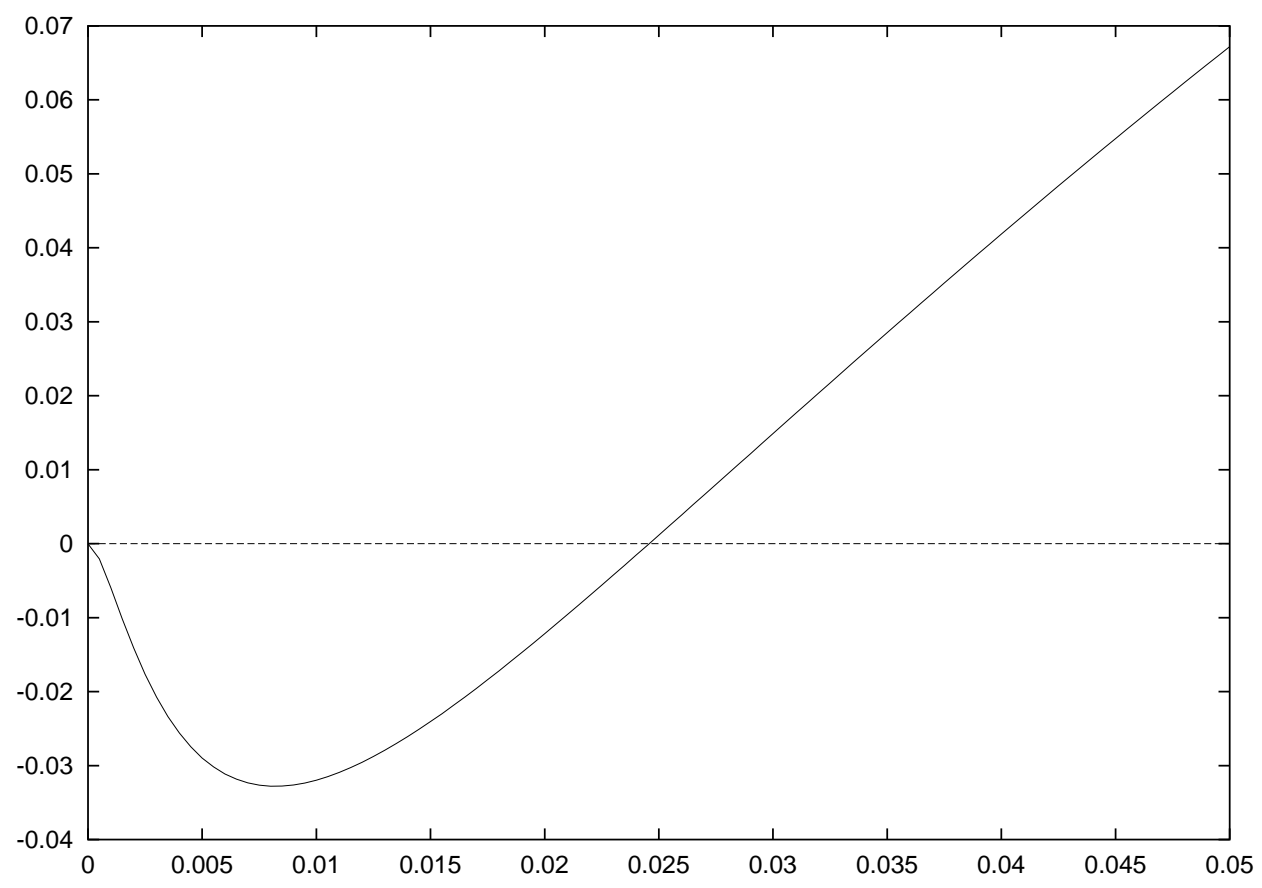

Figure 3: Plot of the Evans function versus $\lambda$ along the real axis, for the case $p=10$.

The equation on $\bigwedge^{2}\left(\mathbb{C}^{5}\right)$

$$
\frac{d}{d x} \widetilde{\mathbf{U}}^{+}=\left[\mathbf{A}^{(2)}(x, \lambda)-\sigma_{+}(\lambda) \mathbf{I}_{d}\right] \widetilde{\mathbf{U}}^{+},\left.\quad \widetilde{\mathbf{U}}^{+}(x, \lambda)\right|_{x=L_{\infty}}=\zeta^{+}(\lambda)
$$

is integrated from $x=L_{\infty}$ to $x=0$, where the scaling

$$
\widetilde{\mathbf{U}}^{+}(x, \lambda)=e^{-\sigma_{+}(\lambda) x} \mathbf{U}^{+}(x, \lambda)
$$

ensures that $\left.\widetilde{\mathbf{U}}^{+}(x, \lambda)\right|_{x=0}$ is bounded. An alternative to this scaling is to impose a renormalization of the vectors during or at the end of the integration, with for example $\left|\widetilde{\mathbf{U}}^{+}(0, \lambda)\right|=1$, but such a scaling does not preserve analyticity. The system (8.6) is integrated using the second-order GL-RK method, i.e. the implicit midpoint method. For a system in the form $\mathbf{U}_{x}=\mathbf{B}(x, \lambda) \mathbf{U}$, each step of the implicit midpoint rule takes the form

$$
\mathbf{U}^{n+1}=\left[\mathbf{I}-\frac{1}{2} \Delta x \mathbf{B}_{n+1 / 2}\right]^{-1}\left[\mathbf{I}+\frac{1}{2} \Delta x \mathbf{B}_{n+1 / 2}\right] \mathbf{U}^{n} \quad \text { where } \quad \mathbf{B}_{n+1 / 2}=\mathbf{B}\left(x_{n+1 / 2}, \lambda\right) .
$$

For $x<0$, the equation

$$
\frac{d}{d x} \tilde{\mathbf{V}}^{-}=\left[-\mathbf{A}^{(2)}(x, \lambda)^{T}+\sigma_{+}(\lambda) \mathbf{I}_{d}\right] \tilde{\mathbf{V}}^{-},\left.\quad \tilde{\mathbf{V}}^{-}(x, \lambda)\right|_{x=-L_{\infty}}=\overline{\eta^{-}(\lambda)},
$$

is integrated from $x=-L_{\infty}$ to $x=0$, also using the implicit midpoint rule, where again we introduce a rescaling

$$
\widetilde{\mathbf{V}}^{-}(x, \lambda)=e^{\sigma_{+}(\lambda) x} \mathbf{V}^{-}(x, \lambda)
$$

to remove the exponential growth. Constant stepsize was used throughout. Predominantly, 20000 steps, $\Delta x=10^{-3}$, were used for each integration, although up to 2000000 steps were used when checking convergence near $p=p_{\text {crit }}$. The numerical accuracy was checked monitoring 


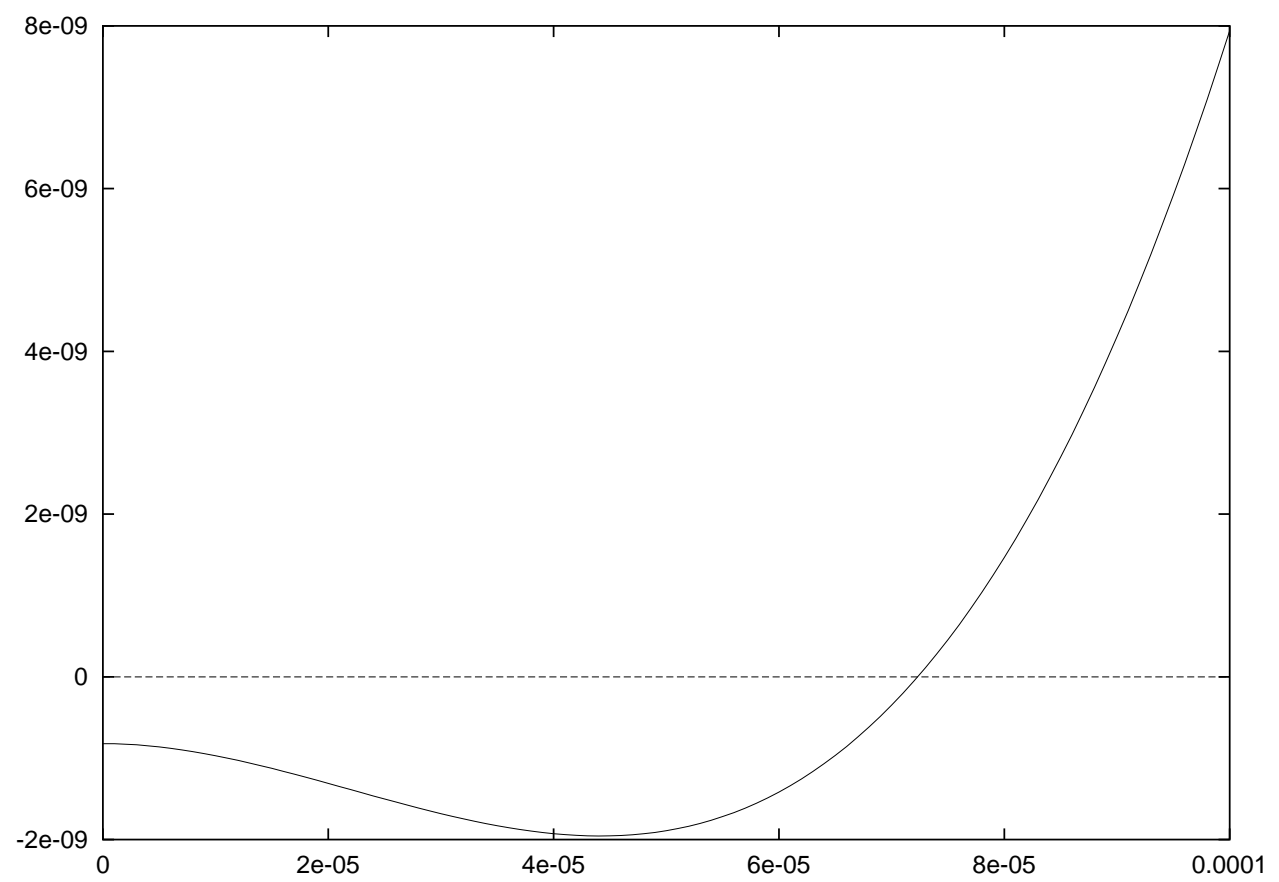

Figure 4: Plot of the Evans function versus $\lambda$ along the real axis, for the linearization about (8.2) near the critical $p$-value with $p=4.8$

the Grassmanian as discussed in Section 5. Values of $I_{1}, \ldots, I_{5}$ were computed, and in the cases checked, they maintained values which were of the order of the truncation error. The algorithm was coded in $\mathrm{C}$, and the programme is freely available from the authors.

At $x=0$ the computed Evans function is

$$
E(\lambda)=\left\langle\overline{\mathbf{V}^{-}(0, \lambda)}, \mathbf{U}^{+}(0, \lambda)\right\rangle_{10}=\left\langle\overline{\widetilde{\mathbf{V}}^{-}(0, \lambda)}, \widetilde{\mathbf{U}}^{+}(0, \lambda)\right\rangle_{10} .
$$

The above calculations confirm a change of stability of a purely real eigenvalue (see Figure 4). However there may be complex eigenvalues. To determine if there are any eigenvalues in the right-half plane with nonzero imaginary part, we use a numerical implementation of Cauchy's Theorem (cf. YING \& KATZ [48], and references therein). The Evans function is evaluated along a line in the complex $\lambda$ plane with constant small real part,

$$
E(\varepsilon+\mathrm{i} y), \quad-Y_{\infty}<y<Y_{\infty}, \quad \varepsilon<<1 .
$$

The small offset $\varepsilon$ is need to circumvent the second-order pole of $E(\lambda)$ at $\lambda=0$. The image of this function is then plotted in the complex $E(\lambda)$ plane. Typically, $Y_{\infty}$ was taken to be $Y_{\infty}=10^{8}$ The number of times that this image encircles the origin is equal to the number of zeros of $E(\lambda)$ in the right-half complex $\lambda$ plane. Results for the cases $p=4$ and $p=10$ are shown in Figure 5 and Figure 6 respectively. For $p=10$ it is clearly seen that the winding number is 1 corresponding to the one root of $E(\lambda)$ for real $\lambda$. To see that for $p=4$ the winding number is 0 , we show in Figure 7 a close-up of the Evans function for $p=4$ near the origin $\lambda=0$ which shows nicely how the winding number behaves for the stable case. In the stable case the origin is outside the big closed loop shown in Fig 5. As a matter of fact if $p$ increases towards the critical $p=p_{\text {crit }}$, the origin moves closer to the circle to enter the closed loop for $p>p_{\text {crit }}$. These figures show that the only unstable eigenvalue for these cases is the real eigenvalue found in Figure 2 and Figure 3. 


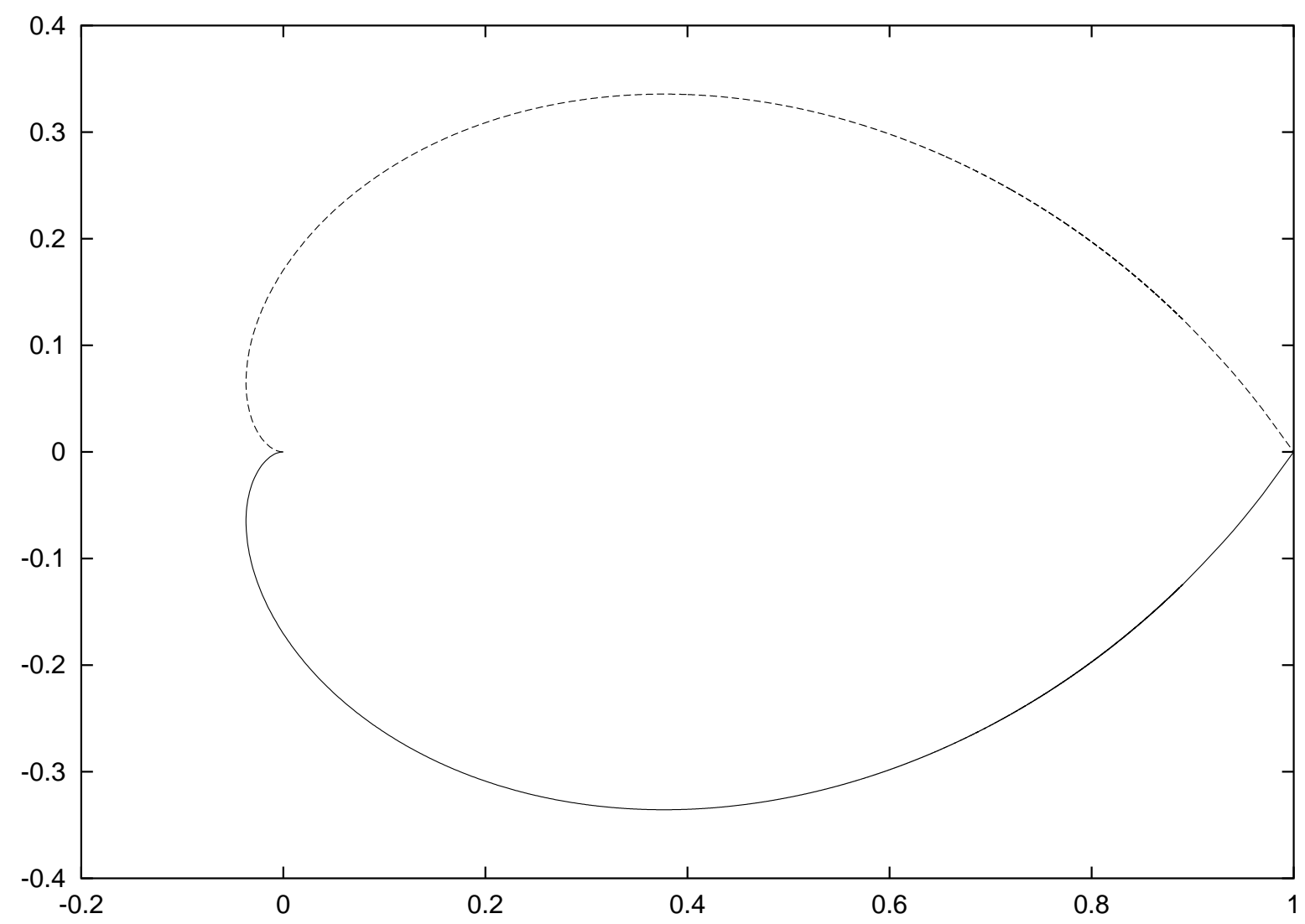

Figure 5: Image of the Evans function in the complex $E(\lambda)$ plane for the case $p=4$.

\section{Concluding remarks}

The stability problem considered in this paper is sometimes called the longitudinal stability problem. The class of perturbations is parallel to the direction of the basic state, in contrast to transverse stability and instability, which correspond to a class of perturbations which travels in an oblique direction. The numerical framework presented here should extend to include the case of transverse instabilities of the 5th-order KdV.

Suppose, for example, that the relevant model equation associated with extension of the 5thorder KdV equation is of KP type. In this case, Haragus-Courcelle \& Ill'ichev [31] show that the extension to two space dimensions of 5th-order KdV takes the form

$$
\left(u_{t}+u u_{x}+\alpha u_{x x x}+\beta u_{x x x x x}\right)_{x}+\sigma u_{y y}=0,
$$

which can be generalized by replacing $u u_{x}$ by $-f\left(u, u_{x}, u_{x x}\right)_{x}$. Suppose there exists a solitary wave state of the form, $u(x, y, t)=\hat{u}(x-c t)$. Linearizing about this state, and taking the spectral ansatz,

$$
\hat{v}(x, y, t)=\Re\left(v(x) \mathrm{e}^{(\lambda t+i \kappa y)}\right),
$$

where $\kappa$ is the transverse wavenumber, leads to the following 6th-order equation

$$
\beta u_{x x x x x x}+\alpha u_{x x x x}+(\hat{u}(x) u)_{x x}-c u_{x x}+\lambda u_{x}-\sigma \kappa^{2} u=0 .
$$




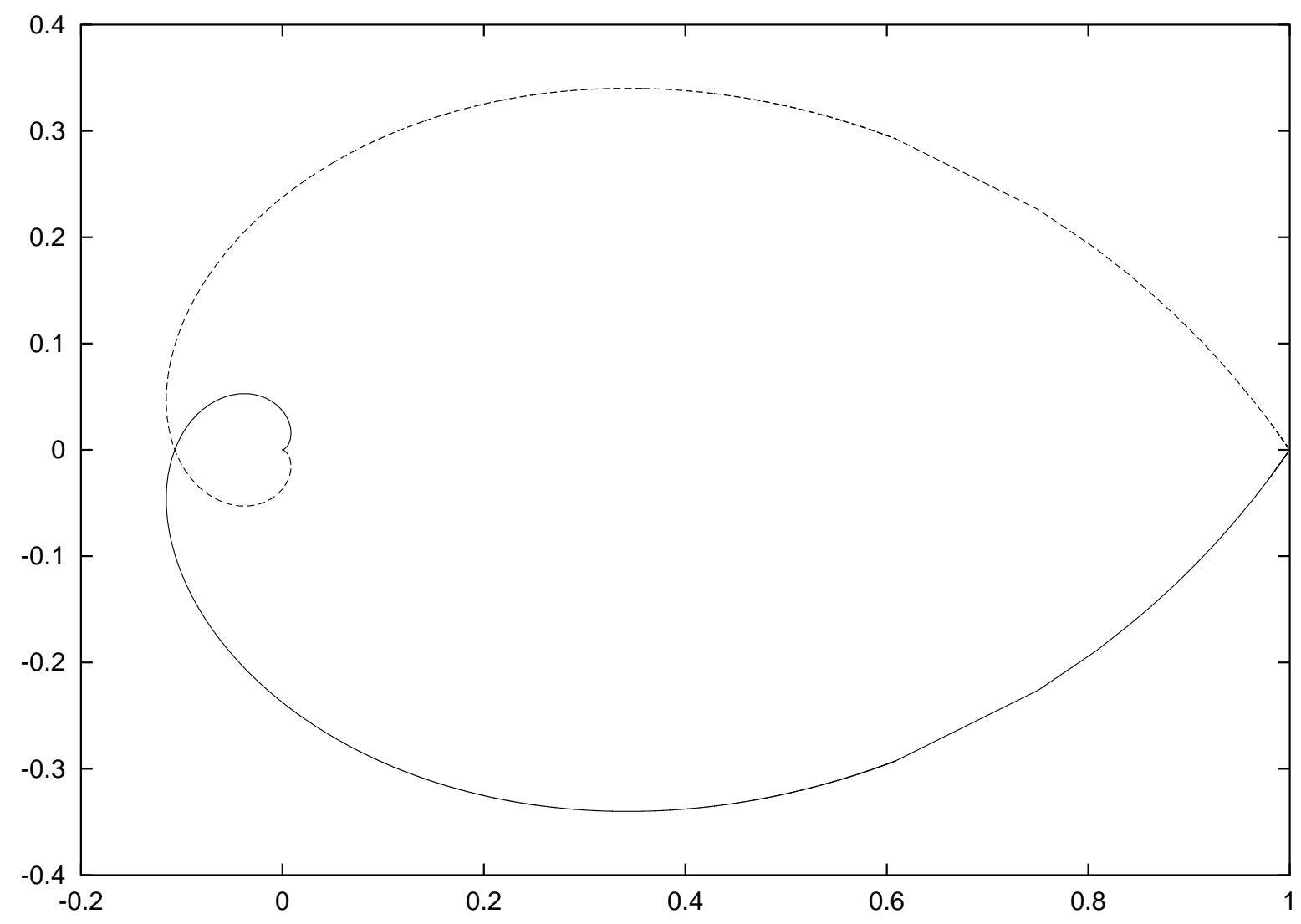

Figure 6: Image of the Evans function in the complex $E(\lambda)$ plane for the case $p=10$.

This equation can be written as a first order system of the form

$$
\mathbf{v}_{x}=\mathbf{A}(x, \lambda, \kappa) \mathbf{v}, \quad \mathbf{v} \in \mathbb{C}^{6} .
$$

With straightforward hypotheses, a $\kappa$-dependent Evans function can be defined for this system, and for $\kappa>0$, the system at infinity, $\mathbf{A}_{\infty}(\lambda, \kappa)$, can have a $1-5,2-4$ or $3-3$ splitting, depending on the values of $\alpha, \beta, c$ and $\sigma$. Therefore the numerical framework in this paper will carry over to this case with obvious generalization: i.e. instead of $\Lambda^{2}\left(\mathbb{C}^{5}\right)$, the system will be integrated on $\bigwedge^{1}\left(\mathbb{C}^{6}\right), \bigwedge^{2}\left(\mathbb{C}^{6}\right)$ or $\bigwedge^{3}\left(\mathbb{C}^{6}\right)$, depending on the splitting at infinity.

A comprehensive study of the transverse instability problem (9.13) has never been considered and would be of great interest. The only known result on transverse instability is for the KdV solitary wave (taking $\beta=0$ and $\alpha=1$ in (9.12)), which is known to be transverse unstable when $\sigma=-1$ and transverse stable when $\sigma=+1$ (cf. Ablowitz \& Segur [1]).

Finally, we mention that neither symmetry or structure of the equations, such as reversibility, symplecticity or multi-symplecticity, has been taken into account in this paper. When such properties are a central part of the equation, it is natural to design the numerical method to respect the structure, and such considerations may lead to new or improved numerical methods.

\section{Acknowledgements}

This research was partially supported by a European Commission Grant, contract number HPRNCT-2000-00113, for the Research Training Network Mechanics and Symmetry in Europe (MASIE), 


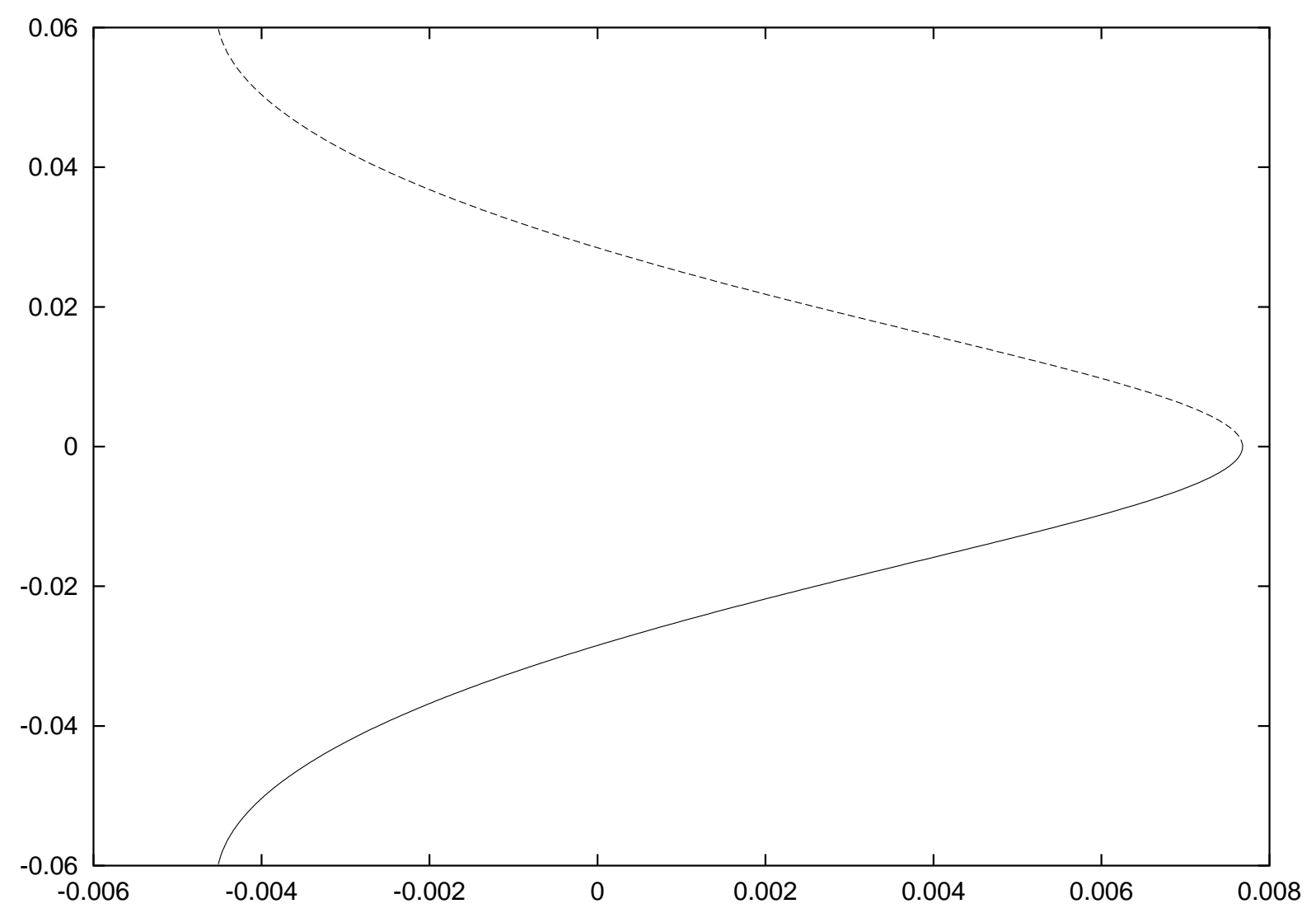

Figure 7: Close-up of the Evans function near $\lambda=0$ in the complex $E(\lambda)$ plane for the case $p=4$.

http://www.inln.cnrs.fr/ montaldi/MASIE/

\section{References}

[1] M.J. Ablowitz \& H. Segur. On the evolution of packets of water waves, J. Fluid Mech. 92, 691-715 (1979).

[2] A.L. Afendikov \& T.J. Bridges. Instability of the Hocking-Stewartson pulse and its implications for three-dimensional Poiseuille flow, Proc. R. Soc. Lond. A 457, 257-272 (2001).

[3] J. Alexander, R. Gardner \& C.K.R.T. Jones. A topological invariant arising in the stability analysis of traveling waves, J. Reine Angew. Math. 410, 167-212 (1990).

[4] J.C. Alexander \& R. SAChs. Linear instability of solitary waves of a Boussinesq-type equation: a computer assisted computation, Nonlin. World 2, 471-507 (1995).

[5] L. Allen \& T.J. Bridges. Numerical exterior algebra and the compound matrix method, Numerische Mathematik (in press, 2002). Electronically published at the website:

http://link.springer.de/link/service/journals/00211/first/bibs/s002110100365.htm

[6] U.M. Ascher, H. Chin \& S. ReICH. Stabilization of DAEs and invariant manifolds, Numerische Mathematik 67, 131-149 (1994). 
[7] I.V. Barashenkov, D.E. Pelinovsky \& E.V. Zemlyanaya. Vibrations and oscillatory instabilities of gap solitons, Phys. Rev. Lett. 80, 5117-5120 (1998).

[8] I.V. Barashenkov \& E.V. Zemlyanaya. Oscillatory instabilities of gap solitons: a numerical study, Comp. Phys. Comm. 126, 22-27 (2000).

[9] S. Benzoni-Gavage, D. Serre \& K. Zumbrun. Alternate Evans functions and viscous shock waves, SIAM J. Math. Anal. 32, 929-962 (2001).

[10] W.-J. Beyn \& J. Lorenz. Stability of traveling waves: dichotomies and eigenvalue conditions on finite intervals, Numer. Func. Anal. Optim. 20, 201-244 (1999).

[11] T.J. Bridges. The Orr-Sommerfeld equation on a manifold, Proc. Roy. Soc. London A 455, 3019-3040 (1999).

[12] T.J. Bridges \& G. Derks. Hodge duality and the Evans function, Phys. Lett. A 251, 363-372 (1999).

[13] T.J. Bridges \& G. Derks. The symplectic Evans matrix, and the linearization about solitary waves and fronts, Arch. Rat. Mech. Anal. 156, 1-87 (2001).

[14] T.J. BRIDGes \& G. DeRks. Linear instability of solitary wave solutions of the Kawahara equation and its generalizations, SIAM J. Math. Anal. (in press, 2002).

[15] T.J. Bridges \& S. Reich. Computing Lyapunov exponents on a Stiefel manifold, Physica D 156, 219-238 (2001).

[16] L.Q. BRIN. Numerical testing of the stability of viscous shock waves, Math. Comp. 70, 10711088 (2001).

[17] L.Q. Brin \& K. Zumbrun. Analytically varying eigenvectors and the stability of viscous shock waves, Preprint, Indiana University (2002).

[18] A.R. Champneys. Homoclinic orbits in reversible systems and their applications in mechanics, fluids and optics, Physica D 112, 158-186 (1998).

[19] A.R. Champneys \& M. Groves. A global investigation of solitary-wave solutions to a two-parameter model for water waves, J. Fluid Mech. 342, 199-229 (1997).

[20] E.A. Coddington \& N. Levinson. Theory of Ordinary Differential Equations, McGrawHill: New York (1955).

[21] G.J. Cooper. Stability of Runge-Kutta methods for trajectory problems, IMA J. Num. Anal. 7 1-13 (1987).

[22] W. Craig \& M.D. Groves. Hamiltonian long-wave approximations to the water-wave problem, Wave Motion 19, 367-389 (1994).

[23] B. Dey, A. Khare \& C.N. Kumar. Stationary solutions of the fifth-order KdV-type equations and their stabilization, Phys. Lett. A223, 449-452 (1996).

[24] F. Dias \& E.A. Kuznetsov. Nonlinear stability of solitons in the fifth-order Korteweg-de Vries equation, Phys. Lett. A 263, 98-104 (1999).

[25] P.G. Drazin \& W.H. ReId. Hydrodynamic stability, Cambridge University Press (1981). 
[26] J.W. Evans. Nerve axon equations IV. The stable and unstable impulse, Indiana Univ. Math. J. 24, 1169-1190 (1975).

[27] J.W. Evans \& J. Feroe. Local stability theory of the nerve impulse, Math. Biosci. 37, 23-50 (1977).

[28] R. Gardner \& K. Zumbrun. The gap lemma and geometric criteria for instability of shock profiles, Comm. Pure Appl. Math. 51, 797-855 (1998).

[29] W.J.F. GovaerTs. Numerical Methods for Bifurcations of Dynamical Equilibria, SIAM Publications: Philadelphia (2000).

[30] M.D. Groves. Solitary-wave solutions to a class of fifth-order model equations, Nonlinearity $11341-353$ (1998).

[31] M. Haragus-Courcelle \& A. Ill'ichev. Three-dimensional solitary waves in the presence of additional surface effects, Euro. J. Mech. B/Fluids 17, 739-768 (1998).

[32] J. HARRIS. Algebraic Geometry, Springer-Verlag: Berlin (1992).

[33] A.T. Ill'IChev \& A.Y. Semenov. Stability of solitary waves in dispersive media described by a fifth-order evolution equation, Theor. Comp. Fluid Dyn. 3, pp.307-326 (1992).

[34] T. Kapitula \& B. Sandstede. Stability for bright solitary wave solutions to perturbed nonlinear Schrödinger equations, PhysicaD 124, 58-103 (1998).

[35] V.I. Karpman. Stabilization of soliton instabilities by higher-order dispersion: KdV-type equations, Phys. Lett. A210, 77-84 (1996).

[36] T. Kato. Perturbation Theory for Linear Operators. Second Edition, Springer Verlag: Heidelberg (1984).

[37] R. Kawahara. Oscillatory solitary waves in dispersive media, J. Phys. Soc. Japan 33, 260264 (1972).

[38] H.B. Keller. Numerical Solution of Two-Point Boundary-Value Problems. CBMS-NSF Conference Series 24, SIAM: Philadelphia (1974).

[39] S. Kichenassamy \& P.J. Olver. Existence and nonexistence of solitary wave solutions to higher order model evolution equations, SIAM J. Math. Anal. 23, 1141-1166 (1992).

[40] S.P. Levandosky. A stability analysis for fifth-order water-wave models, PhysicaD 125, 222-240 (1999).

[41] M. Liefvendahl \& G. Kreiss. Numerical investigation of examples of unstable viscous shock waves, Proceedings of Hyp2000 (8th Inter. Conf. on Hyper. Problems), Universität Magdeburg (2000).

[42] M. Marcus. Finite Dimensional Multilinear Algebra, Part II, Marcel Dekker: New York (1975).

[43] J.R. Magnus \& H. Neudecker, Matrix Differential Calculus, John Wiley \& Sons, 1988.

[44] B.S. NG \& W.H. ReID. An initial-value method for eigenvalue problems using compound matrices, J. Comp. Phys. 30, 125-136 (1979). 
[45] R.L. Pego, P. Smereka \& M.I. Weinstein. Oscillatory instability of traveling waves for a KdV-Burgers equation, PhysicaD 67, 45-65 (1993).

[46] A. Soffer \& M.I. Weinstein. Resonances, radiation damping and instability in Hamiltonian nonlinear wave equations, Inven. Math. 136, 9-74 (1999).

[47] J. Swinton \& J. Elgin. Stability of travelling pulse to a laser equation, Phys. Lett. A 145, 428-433 (1990).

[48] X. Ying \& I.N. Katz. A reliable argument principle algorithm to find the number of zeros of an analytic function in a bounded domain, Numer. Math. 53, 143-163 (1988).

[49] R.O. Wells. Differential Analysis on Complex Manifolds, Springer-Verlag: Berlin (1980). 